\title{
Electronic behavioral interventions for headache: a systematic review
}

Mia Tova Minen ${ }^{1,2^{*}}$, John Torous ${ }^{3}$, Jenelle Raynowska ${ }^{4}$, Allison Piazza ${ }^{5}$, Corita Grudzen $^{6}$, Scott Powers ${ }^{7}$, Richard Lipton ${ }^{8}$ and Mary Ann Sevick ${ }^{9}$

\begin{abstract}
Background: There is increasing interest in using electronic behavioral interventions as well as mobile technologies such as smartphones for improving the care of chronic disabling diseases such as migraines. However, less is known about the current clinical evidence for the feasibility and effectiveness of such behavioral interventions.

Objective: To review the published literature of behavioral interventions for primary headache disorders delivered by electronic means suitable for use outside of the clinician's office.

Methods: An electronic database search of PubMed, PsycINFO, and Embase was conducted through December 11, 2015. All eligible studies were systematically reviewed to examine the modality in which treatment was delivered (computer, smartphone, watch and other), types of behavioral intervention delivered (cognitive behavioral therapy [CBT], biofeedback, relaxation, other), the headache type being treated, duration of treatment, adherence, and outcomes obtained by the trials to examine the overall feasibility of electronic behavioral interventions for headache.

Results: Our search produced 291 results from which 23 eligible articles were identified. Fourteen studies used the internet via the computer, 2 used Personal Digital Assistants, 2 used CD ROM and 5 used other types of devices. None used smartphones or wearable devices. Four were pilot studies $(N \leq 10)$ which assessed feasibility. For the behavioral intervention, CBT was used in 11 (48\%) of the studies, relaxation was used in 8 (35\%) of the studies, and biofeedback was used in $5(22 \%)$ of the studies. The majority of studies $(14 / 23,61 \%)$ used more than one type of behavioral modality. The duration of therapy ranged from 4-8 weeks for CBT with a mean of 5.9 weeks. The duration of other behavioral interventions ranged from 4 days to 60 months. Outcomes measured varied widely across the individual studies.
\end{abstract}

Conclusions: Despite the move toward individualized medicine and mHealth, the current literature shows that most studies using electronic behavioral intervention for the treatment of headache did not use mobile devices. The studies examining mobile devices showed that the behavioral interventions that employed them were acceptable to patients. Data are limited on the dose required, long term efficacy, and issues related to the security and privacy of this health data.

This study was registered at the PROSPERO International Prospective Register of Systematic Reviews (CRD42015032284) (Prospero, 2015).

Keywords: Migraine, Headache, Behavioral medicine, Cognitive behavioral therapy, Biofeedback, Progressive muscle relaxation therapy, Electronic

\footnotetext{
* Correspondence: minenmd@gmail.com

'Department of Neurology, NYU Langone Medical Center, 240 East 38th

Street 20th floor, New York, NY 10016, USA

${ }^{2} \mathrm{NYU}$ Langone Headache Center, Department of Neurology, NYU School of

Medicine, New York, NY, USA

Full list of author information is available at the end of the article
}

\section{Springer}

(c) 2016 Minen et al. Open Access This article is distributed under the terms of the Creative Commons Attribution 4.0 International License (http://creativecommons.org/licenses/by/4.0/), which permits unrestricted use, distribution, and reproduction in any medium, provided you give appropriate credit to the original author(s) and the source, provide a link to the Creative Commons license, and indicate if changes were made. 


\section{Introduction}

Experts in behavioral headache medicine have identified ten areas of critical need for behavioral headache research. One important unmet need is the development of an innovative technology-based treatment platform for headache self-management [1]. Behavioral headache treatments (e.g., progressive muscle relaxation (PMR), biofeedback, and cognitive-behavioral therapy (CBT)) are Level A Evidence-Based migraine treatments [2] that are essentially free of side effects [3]. Behavioral treatments have enduring benefits [4] and may be less costly than pharmacologic interventions [5]. Questions remain whether these evidence-based treatments can effectively be delivered electronically so that patients can do them on their own outside of the clinical setting. This is an important and timely topic because in 2014, 64 million Americans had smartphones [6]. The FDA states that per industry estimates, 500 million smartphone users worldwide will be using a health care application (app) by 2015 [7], and by 2018, 50 percent of the more than 3.4 billion smartphone and tablet users will have downloaded mobile health apps [8]. Most published literature regarding health apps has focused on preventing and managing chronic disease $[9,10]$, monitoring app acceptability and utility [11-14], and qualitative studies of user experience and desired functions [15-18]. However, it is unclear the extent to which apps are effective at facilitating behavior change [19].

The creation of electronic headache apps has proliferated, with over 40 headache apps in the U.S. Google Play Store (Date accessed 12/23/15) and over 70 headache apps in the U.S. Apple iTunes store (Date accessed 1/8/ 16). These apps advertise various purposes-the ability to track headache frequency with electronic headache diary functionality, the ability to detect headache triggers, and the ability to treat headaches with behavioral treatments. Prior studies have demonstrated that smartphone apps with electronic headache diaries are a reliable method for data collection preferred over paper headache diaries by patients because electronic diaries are more discreet in the work place [20]. Electronic headache diary data collection also results in fewer secondary data errors [21], less administrative burden [22, 23], high participant acceptance [23], and potential cost savings [24]. Additionally, this format allows the use of reminders and timely follow-up of non-compliant participants via real time investigator data monitoring capabilities. Thus, while we know that electronic headache diaries are useful for collecting data, it is unknown whether behavioral interventions, oftentimes delivered along with the electronic diaries, are feasible and effective.

The purpose of this systematic review is to describe the current body of literature on electronic behavioral interventions for primary headaches that can be used outside of the clinician's office. Specifically, we reviewed the literature to first examine the modalities in which such treatments were delivered (computer, smartphone, watch and other), types of behavioral intervention delivered (CBT, biofeedback, relaxation, other), the headache type being treated, duration of the treatment, adherence, and outcomes obtained by the trials. We also examine the overall feasibility of smartphone interventions for primary headache disorders. Understanding the existing literature on the electronic behavioral interventions will increase our understanding of what has been learnt so far, where the potential of this technology may be best realized in future, and what may be applied to the growing field of mHealth.

\section{Review}

\section{Methods}

A health sciences librarian (AP) conducted searches in PubMed.gov, Embase (via Ovid), and PsycINFO (via Ovid) from January 1, 2000 through December 11, 2015. The search strategy combined three concepts: (1) headaches (e.g. "migraines"), (2) electronic or computerized formats (e.g. "internet"), and (3) behavioral interventions (e.g. "behavior therapy"). Terms were searched both as keywords and Subject Heading terms. The search was restricted to studies published in or after 2000. A manual search among references of selected articles and reviews was also performed (MM). The full search strategies are available in the Appendix. After deduplication, 291 unique records were identified.

Studies were considered eligible for inclusion if they were randomized controlled trials, prospective nonrandomized trials, or observational studies using a behavioral intervention for a primary headache disorder. Secondary headache disorders, including dental disorders, were not eligible. Studies involving telephonic interventions were not included.

Two study investigators (MM, JT) included or excluded articles based on the predefined eligibility criteria using a two-step procedure. In the first step, the investigators independently reviewed titles and abstracts. Twenty-two articles were resolved by consensus, and 256 articles were excluded. Main reasons for exclusion based on title and abstract were because the papers did not pertain to a primary headache disorder $(N=99)$, were review articles $(N$ $=78$ ), or were studies about medications (not behavioral interventions) $(N=25)$.

In the second step, the same investigators independently reviewed the full-text version of the remaining 32 eligible articles. Any differences in selection from the two independent searches were resolved by consensus between the two investigators. Of the 32 remaining articles for full-text screening, 23 met inclusion criteria. Two articles were resolved by consensus. 
A standardized form was used for data extraction, including the following items: first author, year of the study, country where the study was performed, type of behavioral intervention, mode of electronic delivery, study design, recruitment and setting, headache type, participants, outcomes measured, and results.

This study was registered at the PROSPERO International Prospective Register of Systematic Reviews (CRD42015032284) [25]. Reporting of this study conforms to the Preferred Reporting Items for Systematic Reviews and Meta-Analyses (PRISMA) statement [26] and the flow diagram can be found in Fig. 1 .

\section{Results}

As indicated in Table 1, 23 studies met our search criteria, 8 were performed in pediatric populations and the remainder performed in adults. The majority (14/23, $61 \%)$ used the internet to deliver the behavioral electronic intervention. Only 2/23 (9\%) used CD ROMs and 2/23 (9 \%) used Personal Digital Assistants (PDAs). A PDA is a handheld digital device which is similar to its successor of the smartphone but often lacks either the touch screen, connectivity, or ability to download and run native applications that a smartphone can. Five used alternate means but none used smartphone technology. About half (13/23, $57 \%$ ) were randomized controlled trials. For the behavioral intervention, CBT was used in $11 / 23$ (48 \%) (one study used family CBT), relaxation was used in 8/23 (35\%) (half of these studies specified that PMR was used for the relaxation therapy), and biofeedback was used in 5/23 (22\%) of the studies. The majority $(14 / 23,61 \%)$ used more than one type of behavioral modality. Descriptions of the procedures for teaching and/or practicing the CBT were varied. Descriptions ranged from "a CD ROM program designed to give children additional strategies to help manage their headache pain" to references of full CBT procedure modules. The table in the Appendix illustrates the varied descriptions provided in the papers. The duration of therapy ranged from 4-8 weeks for CBT with a mean of 5.9 weeks. The duration of other behavioral interventions ranged from 4 days to 60 months.

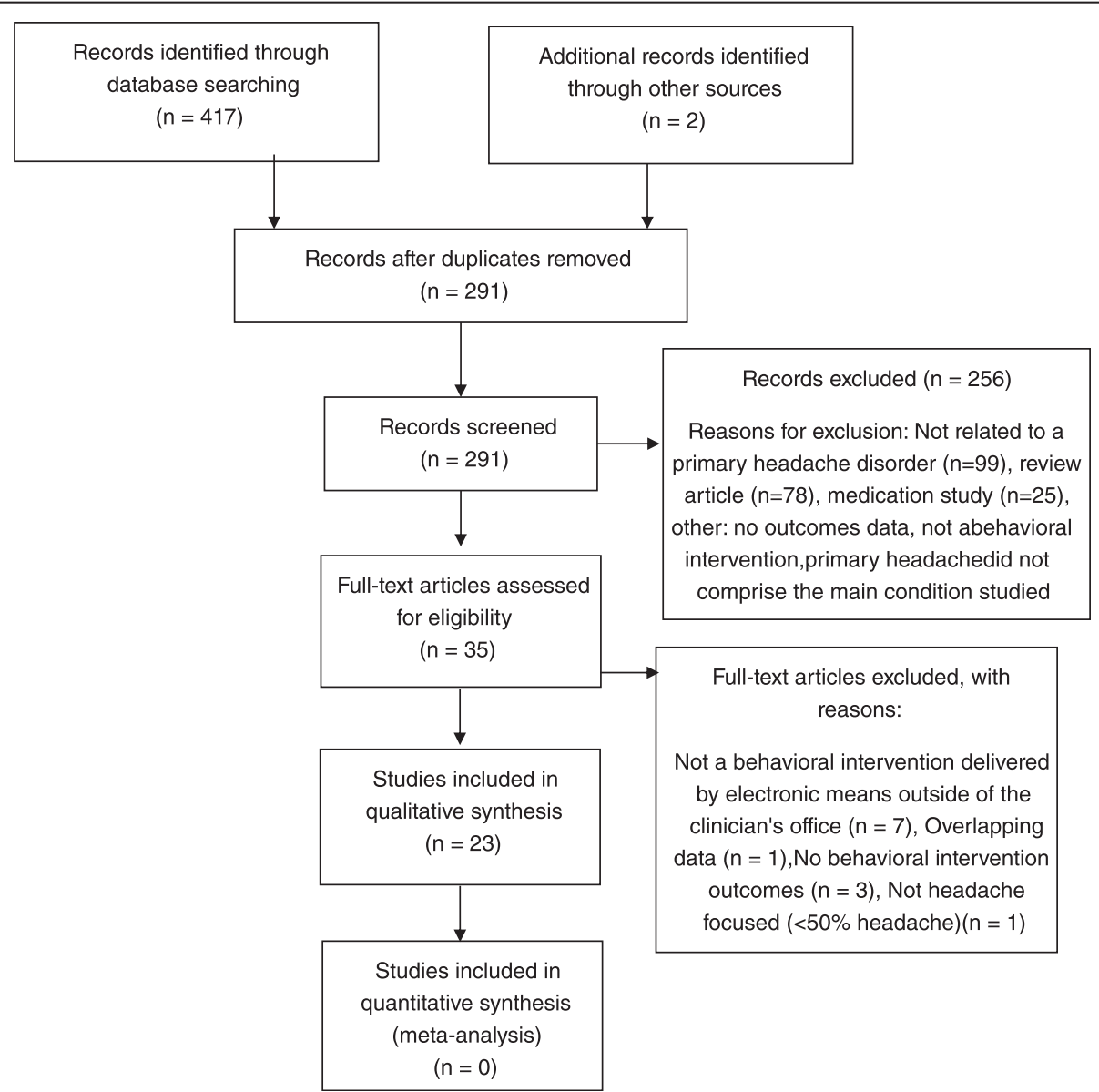

Fig. 1 Preferred reporting items for systematic reviews and meta-analyses (PRISMA) flow diagram 
Table 1 Description of studies meeting eligibility for the systematic review

\begin{tabular}{|c|c|c|c|c|c|c|c|c|c|}
\hline Tool & $\begin{array}{l}\text { Type of } \\
\text { behavioral } \\
\text { intervention }\end{array}$ & Design & $\begin{array}{l}\text { First author, } \\
\text { year }\end{array}$ & $\begin{array}{l}\text { \# of participants } \\
(\mathrm{N}) \text {, setting, } \\
\text { duration of } \\
\text { treatment }\end{array}$ & $\begin{array}{l}\text { Headache } \\
\text { type/criteria }\end{array}$ & $\begin{array}{l}\text { Outcomes } \\
\text { measured }\end{array}$ & $\begin{array}{l}\text { Results (HA freq, HA } \\
\text { intensity, disability, } \\
\text { adherence) }\end{array}$ & Other results & Drop out rate \\
\hline \multirow[t]{8}{*}{ CD ROM } & \multirow[t]{5}{*}{ CBT } & \multirow[t]{5}{*}{$\begin{array}{l}\mathrm{RCT} \text { (waitlist } \\
\text { control) }\end{array}$} & \multirow[t]{5}{*}{$\begin{array}{l}\text { Connelly, } \\
2005 \text { [44] }\end{array}$} & \multirow[t]{5}{*}{$\begin{array}{l}N=50 \text { (ages 7-12) } \\
\text { Pediatric } \\
\text { Neurology Clinic } \\
4 \text { weeks }\end{array}$} & \multirow[t]{5}{*}{$\begin{array}{l}\text { Migraine, tension } \\
\text { type or chronic } \\
\text { daily HA min } \\
4 / \text { month with } \\
\text { symptom free } \\
\text { period, assessed } \\
\text { by neurologist } \\
\text { or NP }\end{array}$} & \multirow[t]{5}{*}{$\begin{array}{l}\text { HA duration; HA } \\
\text { days; HA intensity; } \\
\text { HA severity; } \\
\text { Medication; Self- } \\
\text { efficacy; QoL; } \\
\text { Disability; } \\
\text { Acceptability }\end{array}$} & \multirow{2}{*}{$\begin{array}{l}\text { - There was a } \\
\text { significant reduction } \\
\text { in HA frequency from } \\
\text { baseline to post-tx in } \\
\text { both the tx } \\
\text { (Headstrong) + control } \\
\text { groups (Univariate } \\
\text { ANOVA, } p<0.001 \text { ) with } \\
\text { a trend suggesting a } \\
\text { greater reduction in } \\
\text { the tx group ( } 60.16 \% \\
\text { reduction in tx group } \\
\text { vs. } 45.43 \% \text { reduction } \\
\text { in controls, } p=0.091 \text { ). }\end{array}$} & $\begin{array}{l}\text { - Significant "group by } \\
\text { phase" interaction } \\
\text { effect on the HA } \\
\text { duration variable } \\
(p=0.014) \text { suggesting } \\
\text { that there was } \\
\text { different changes in } \\
\text { HA duration from } \\
\text { baseline to 1-month } \\
\text { post-tx as a function } \\
\text { of group assignment. }\end{array}$ & \multirow[t]{5}{*}{$\begin{array}{l}6 \% \text { Overall } \\
\text { (4\% tx group } \\
\text { vs } 2 \% \text { control } \\
\text { group) }\end{array}$} \\
\hline & & & & & & & & \multirow{4}{*}{$\begin{array}{l}\text { - A clinically } \\
\text { significant change in } \\
\text { Hl from baseline to } \\
1 \text { month post-tx was } \\
\text { observed in } 60 \% \text { of } \\
\text { the tx group + only } \\
8 \% \text { of the control, } \\
\text { therefore using the } \\
\text { adjunctive Head- } \\
\text { strong program re- } \\
\text { sulted in more } \\
\text { children achieving } \\
\text { clinically significant } \\
\text { outcomes (Chi-square, } \\
p=0.005 \text { ) }\end{array}$} & \\
\hline & & & & & & & $\begin{array}{l}\text { - Significant "group by } \\
\text { phase" interaction } \\
\text { effect on the HA } \\
\text { intensity variable } \\
(p=0.004) \text { suggesting } \\
\text { that there was different } \\
\text { changes in HA intensity } \\
\text { from baseline to } \\
\text { 1-month post-tx as a } \\
\text { function of group } \\
\text { assignment. }\end{array}$ & & \\
\hline & & & & & & & $\begin{array}{l}\text { HA intensity decreased } \\
\text { from baseline to } \\
1 \text { month post-tx in the } \\
\text { tx group, while it } \\
\text { remained fairly constant } \\
\text { in the control group. }\end{array}$ & & \\
\hline & & & & & & & $\begin{array}{l}\text { - No power to assess } \\
\text { secondary outcomes } \\
\text { including disability. }\end{array}$ & & \\
\hline & \multirow[t]{3}{*}{$\begin{array}{l}\text { CBT + self- } \\
\text { management }\end{array}$} & \multirow[t]{3}{*}{ RCT } & \multirow[t]{3}{*}{$\begin{array}{l}\text { Rapoff, } 2014 \\
\text { [45] }\end{array}$} & \multirow{3}{*}{$\begin{array}{l}N=35 \text { (ages } 7-12 \text { ) } \\
\text { Pediatric clinics \& } \\
\text { children's hospitals } \\
4 \text { weeks }\end{array}$} & \multirow[t]{3}{*}{$\begin{array}{l}\text { migraine with } \\
\text { or without aura } \\
\text { min } 1 / \text { week }\end{array}$} & \multirow{3}{*}{$\begin{array}{l}\text { HA duration; HA } \\
\text { frequency; HA } \\
\text { days; HA severity; } \\
\text { QoL; Disability }\end{array}$} & $\begin{array}{l}\text { - NS change in HA } \\
\text { frequency between } \\
\text { tx + control groups }\end{array}$ & & \multirow{2}{*}{$\begin{array}{l}50 \% \text { Overall- } \\
\text { no allocated } \\
\text { intervention } \\
\text { ( } 55 \% \text { tx group } \\
\text { vs. } 43.3 \% \\
\text { control group) }\end{array}$} \\
\hline & & & & & & & \multirow{2}{*}{$\begin{array}{l}\text { - There was a } \\
\text { statistically significant } \\
\text { difference in pain } \\
\text { severity (10-point VAS) } \\
\text { post-intervention, with } \\
\text { tx group reporting } \\
\text { lower pain severity } \\
\text { than control group }\end{array}$} & & \\
\hline & & & & & & & & & $\begin{array}{l}18.6 \% \text { Overall } \\
\text { were lost to } \\
\text { follow-up } \\
\text { (17.5\% tx }\end{array}$ \\
\hline
\end{tabular}


Table 1 Description of studies meeting eligibility for the systematic review (Continued)

\begin{tabular}{|c|c|c|c|c|c|c|c|c|c|}
\hline & & & & & & & $\begin{array}{l}(5.06 \text { vs. } 6.25, p=0.03 \\
E S=0.7)\end{array}$ & & $\begin{array}{l}\text { group vs. } 20 \% \\
\text { control group) }\end{array}$ \\
\hline & & & & & & & $\begin{array}{l}\text { - At } 3 \text { months post- } \\
\text { intervention, parents } \\
\text { reported lower } \\
\text { migraine-related } \\
\text { disability (PedMIDAS) } \\
\text { in the tx group } \\
\text { compared to control } \\
\text { group (1.36 vs. } 5.18 \text {, } \\
p=0.04 \text { ). }\end{array}$ & & \\
\hline \multirow[t]{8}{*}{ Internet } & \multirow[t]{4}{*}{ CBT } & \multirow[t]{4}{*}{$\begin{array}{l}\text { Parallel group } \\
\text { unblinded RCT }\end{array}$} & \multirow[t]{4}{*}{$\begin{array}{l}\text { Day, } 2014 \\
\text { [46] }\end{array}$} & \multirow{4}{*}{$\begin{array}{l}N=36 \text { (ages 19+) } \\
\text { Physician referral, } \\
\text { brochures }+ \\
\text { public service } \\
\text { announcements } \\
7 \text { weeks }\end{array}$} & \multirow{4}{*}{$\begin{array}{l}\text { Migraine, Tension- } \\
\text { type, cluster or } \\
\text { other primary HA } \\
\text { min } 3 \text { days/month }\end{array}$} & \multirow{4}{*}{$\begin{array}{l}\text { HA duration; HA } \\
\text { index; HA } \\
\text { frequency; HA } \\
\text { intensity; HA } \\
\text { severity; } \\
\text { Medication; Self- } \\
\text { efficacy; Disability; } \\
\text { Acceptability; } \\
\text { Alliance; Feasibility; } \\
\text { Engagement; } \\
\text { Other }\end{array}$} & \multirow{3}{*}{$\begin{array}{l}\text { - There was a } \\
\text { statistically significant } \\
\text { baseline to post-test } \\
\text { decrease in HA } \\
\text { frequency, HA peak } \\
\text { intensity + HA average } \\
\text { intensity in the total } \\
\text { completer sample, } \\
\text { however there were } \\
\text { no significant } \\
\text { differences in these } \\
\text { variables between the } \\
\text { tx + control groups. }\end{array}$} & \multirow{2}{*}{$\begin{array}{l}\text { - ITT analysis: Greater } \\
\text { improvement in self- } \\
\text { efficacy }(p=0.02)+ \\
\text { pain acceptance } \\
(p=0.02) \text { in tx group } \\
\text { compared to controls. }\end{array}$} & \multirow{3}{*}{$\begin{array}{l}11.3 \% \text { Overall } \\
\text { prior to } \\
\text { randomization } \\
2.1 \% \text { Overall } \\
\text { after } \\
\text { randomization } \\
\text { (.53\% in tx } \\
\text { group vs. } \\
1.6 \% \text { in } \\
\text { control group) }\end{array}$} \\
\hline & & & & & & & & & \\
\hline & & & & & & & & $\begin{array}{l}\text { - Completer analysis: } \\
\text { Improved pain } \\
\text { interference } \\
(p<0.01)+\text { pain } \\
\text { catastrophizing } \\
(p=0.03) \text { in tx group } \\
\text { compared to controls. }\end{array}$ & \\
\hline & & & & & & & $\begin{array}{l}\text { - For the ITT analysis, } \\
\text { there was a significant } \\
\text { decrease in HA } \\
\text { frequency overall, but } \\
\text { again no difference in } \\
\text { tx groups. }\end{array}$ & $\begin{array}{l}\text { - MBCT was found to } \\
\text { be feasible, tolerable + } \\
\text { acceptable to patients. }\end{array}$ & \\
\hline & \multirow[t]{4}{*}{ CBT } & \multirow[t]{4}{*}{$\begin{array}{l}\text { Prospective } \\
\text { parallel group } \\
\text { design }\end{array}$} & \multirow[t]{4}{*}{$\begin{array}{l}\text { Bromberg, } \\
2012[27]\end{array}$} & \multirow{4}{*}{$\begin{array}{l}N=213 \text { (ages } \\
18-65 \text { ) Website } \\
\text { postings, } \\
\text { electronic } \\
\text { newsletter } \\
\text { announcements, } \\
\text { neurology clinics, } \\
+ \\
\text { social networking/ } \\
\text { community sites } \\
4 \text { weeks }\end{array}$} & \multirow[t]{4}{*}{$\begin{array}{l}\text { migraine with or } \\
\text { without aura min } \\
2 / \text { month }\end{array}$} & \multirow{4}{*}{$\begin{array}{l}\text { Pain } \\
\text { catastrophizing; } \\
\text { Self-efficacy; } \\
\text { Disability; LoC; } \\
\text { CPC; DAS; Other }\end{array}$} & \multirow{4}{*}{$\begin{array}{l}\text { - Reduction of HA } \\
\text { frequency + severity } \\
\text { could not be tested } \\
\text { due to technical } \\
\text { problems resulting in } \\
\text { loss of data. } \\
\text { - Both tx + control } \\
\text { subjects reported } \\
\text { similar reductions in } \\
\text { disability on MIDAS } \\
\text { (12.8\% decrease + } \\
13.0 \% \text { decrease } \\
\text { respectively) } \\
\text { immediately post- } \\
\text { intervention. }\end{array}$} & \multirow{3}{*}{$\begin{array}{l}\text { - Decrease in } \\
\text { depression (DASS) in } \\
\text { tx group compared } \\
\text { to controls from } \\
\text { baseline to 3-months } \\
\text { post-intervention } \\
(p=0.0009)+\text { baseline } \\
\text { to 6-months } \\
\text { post-intervention } \\
(p=0.0079) .\end{array}$} & \multirow{4}{*}{$\begin{array}{l}11.3 \% \text { Overall } \\
\text { prior to } \\
\text { randomization } \\
2.1 \% \text { Overall } \\
\text { after } \\
\text { randomization } \\
\text { (.53\% in tx } \\
\text { group vs. } \\
1.6 \% \text { in } \\
\text { control group) }\end{array}$} \\
\hline & & & & & & & & & \\
\hline & & & & & & & & & \\
\hline & & & & & & & & $\begin{array}{l}\text { - Decrease in stress } \\
\text { (DASS) in tx group } \\
\text { compared to controls } \\
\text { from baseline to } \\
\text { post-intervention } \\
(p=0.0324)+\text { from } \\
\text { baseline to } 3 \text { month } \\
\text { follow-up ( } p=0.0045) \text {. }\end{array}$ & \\
\hline
\end{tabular}


Table 1 Description of studies meeting eligibility for the systematic review (Continued)

\begin{tabular}{|c|c|c|c|c|}
\hline $\begin{array}{l}\text { CBT (family- } \\
\text { based) }\end{array}$ & RCT & $\begin{array}{l}\text { Law, } 2015 \\
\text { [43] }\end{array}$ & $\begin{array}{l}N=83 \text { (ages } \\
11-17 \text { ) Pediatric } \\
\text { clinic } 8 \text { weeks }\end{array}$ & $\begin{array}{l}\text { Recurrent HA } \\
\text { (>3 months) }\end{array}$ \\
\hline
\end{tabular}

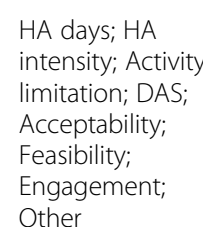

Other
- Follow-up assessment completion in tx vs. control groups respectively were $80 \%$ vs. $89 \%$ at 1month, $70 \%$ vs. $82 \%$ at 3-months, $+55 \%$ vs. $82 \%$ at 6 -months.

- There was a statistically significant reduction in HA frequency from baseline to post-tx + baseline to 3-month follow up in both tx conditions, however there was NS

difference in $\mathrm{HA}$ frequency between tx + control groups.

- There was a statistically significant reduction in HA pain intensity from baseline to post-tx + baseline to
- Reduction in pain

catastrophizing (PCS) in

tx group compared to

controls from baseline

to post intervention

$(p=0.0030)$, 3-month

follow up $(p=0.0099)$,

$(p=0.0006)$.

- CPCI-42:Increase in relaxation (baseline to

post-intervention, 3-

month assessment

6 month assessment)

task persistence

(baseline to post-

intervention + 3-

month assessment),

exercising (baseline to

post-intervention) + use

of social support

(baseline to post-

intervention) in tx

group compared to

controls.

- Increases in self-

efficacy in tx group

compared to controls

(baseline to post-

intervention, 3-month

assessment +6 month assessment)

- There was a

$28.9 \%$ Overall

significant reduction in $(29.5 \%$ in tx

activity limitations, group vs

emotional functioning $28.2 \%$ in

+ parent response to control group

pain behavior from

significant but no

groups 
Table 1 Description of studies meeting eligibility for the systematic review (Continued)

\begin{tabular}{|c|c|c|c|c|c|c|c|c|}
\hline \multirow[t]{3}{*}{$\mathrm{CBT}+\mathrm{PMR}$} & \multirow[t]{3}{*}{$\mathrm{RCT}$} & \multirow[t]{3}{*}{$\begin{array}{l}\text { Trautmann, } \\
2010 \text { [47] }\end{array}$} & \multirow[t]{3}{*}{$\begin{array}{l}N=65 \text { (ages } \\
10-18 \text { ) Newspaper } \\
\text { ads, websites } \\
6 \text { weeks }\end{array}$} & \multirow[t]{3}{*}{$\begin{array}{l}\text { Migraine, tension- } \\
\text { type HA, or } \\
\text { combined HA } \\
\text { min } 2 \mathrm{HA} \\
\text { attacks/month }\end{array}$} & \multirow[t]{3}{*}{$\begin{array}{l}\text { HA duration; HA } \\
\text { frequency; HA } \\
\text { intensity; Pain } \\
\text { catastrophizing; } \\
\text { QoL; DAS; } \\
\text { Acceptability; } \\
\text { Alliance; Other }\end{array}$} & $\begin{array}{l}\text { - There was a } \\
\text { significant reduction } \\
\text { in HA frequency + } \\
\text { duration post-tx in all } \\
\text { groups, but NS } \\
\text { between groups. }\end{array}$ & $\begin{array}{l}\text { - Pain catastrophizing } \\
\text { was significantly } \\
\text { reduced post- } \\
\text { assessment in all } \\
\text { groups, but no } \\
\text { difference was found } \\
\text { between groups. }\end{array}$ & \multirow[t]{3}{*}{$\begin{array}{l}7.7 \% \text { Overall } \\
\text { (16.6\% in CBT } \\
\text { group vs. } 0 \% \\
\text { in AR group } \\
\text { vs. } 5.3 \% \text { in } \\
\text { EDU group) }\end{array}$} \\
\hline & & & & & & $\begin{array}{l}\text { - No significant } \\
\text { difference in HA } \\
\text { intensity was found in } \\
\text { any group at post- } \\
\text { assessment }\end{array}$ & \multirow{2}{*}{$\begin{array}{l}\text { - Responder rates } \\
\text { (reduction in HA } \\
\text { frequency of } 50 \% \text { or } \\
\text { more from baseline) } \\
\text { were significantly } \\
\text { higher in CBT ( } 63 \%) \\
+ \text { AR }(32 \%) \text { groups, } \\
\text { compared to the } \\
\text { EDU/ control group } \\
(19 \%) \text {.This resulted in } \\
\text { NNTs of } 2.0 \text { for CBT + } \\
5.2 \text { for AR. }\end{array}$} & \\
\hline & & & & & & $\begin{array}{l}\text { - There was no } \\
\text { significant difference } \\
\text { in depression, } \\
\text { psychopathological } \\
\text { symptoms, + health- } \\
\text { related quality of life } \\
\text { in any group post- } \\
\text { assessment. }\end{array}$ & & \\
\hline \multirow[t]{3}{*}{$\begin{array}{l}\text { CBT + } \\
\text { Relaxation }\end{array}$} & \multirow[t]{3}{*}{ RCT } & \multirow[t]{3}{*}{$\begin{array}{l}\text { Sorbi, } 2015 \\
{[48]}\end{array}$} & \multirow[t]{3}{*}{$\begin{array}{l}N=368(18-65) \\
\text { HA } \\
\text { centers, website, + } \\
\text { flyers } 8 \text { weeks* }\end{array}$} & \multirow[t]{3}{*}{$\begin{array}{l}\text { Migraine with } \\
2-6 \text { attacks in } \\
\text { the month prior } \\
\text { to randomization }\end{array}$} & \multirow[t]{3}{*}{$\begin{array}{l}\text { HA index; HA } \\
\text { intensity; } \\
\text { Medication; Self- } \\
\text { efficacy; QoL; Dis- } \\
\text { ability; LoC; Other }\end{array}$} & \multirow[t]{3}{*}{$\begin{array}{l}\text { - NS in HA frequency } \\
\text { or intensity in either } \\
\text { group or between } \\
\text { groups. }\end{array}$} & $\begin{array}{l}\text { - HA duration } \\
\text { decreased } \\
\text { significantly more in } \\
\text { telephone arm ( } p< \\
0.05)\end{array}$ & \multirow[t]{3}{*}{$\begin{array}{l}32 \% \text { Overall } \\
\text { ( } 29 \% \text { tx vs. } \\
35 \% \text { control) }\end{array}$} \\
\hline & & & & & & & $\begin{array}{l}\text { - NS in HI between } \\
\text { groups }\end{array}$ & \\
\hline & & & & & & & $\begin{array}{l}\text { - Self-reported } \\
\text { inventories (HADS } \\
\text { depression subscale, } \\
\text { HDI, PSS) showed } \\
\text { significant } \\
\text { improvements in } \\
\text { both groups but not } \\
\text { between groups. }\end{array}$ & \\
\hline $\begin{array}{l}\text { Multimodal } \\
\text { including CBT }\end{array}$ & RCT & $\begin{array}{l}\text { Trautmann, } \\
2008 \text { [49] }\end{array}$ & $\begin{array}{l}N=18 \text { (ages 10- } \\
\text { 18) Participation } \\
\text { was online, } \\
\text { recruitment } \\
\text { strategy not } \\
\text { specified } 6 \text { weeks }\end{array}$ & $\begin{array}{l}\text { Migraine +/or } \\
\text { tension-type } \\
\text { HA min } 2 \text { HA } \\
\text { attacks/ month }\end{array}$ & $\begin{array}{l}\text { HA duration; HA } \\
\text { frequency; HA } \\
\text { intensity; Pain } \\
\text { catastrophizing; } \\
\text { Acceptability; } \\
\text { Alliance }\end{array}$ & $\begin{array}{l}\text { - No significant } \\
\text { difference found } \\
\text { between HA } \\
\text { frequency or intensity } \\
\text { between groups post- } \\
\text { tx. }\end{array}$ & $\begin{array}{l}\text { - NS found between } \\
\text { the two groups post tx } \\
\text { in any of the outcome } \\
\text { variables (HA } \\
\text { frequency, intensity, } \\
\text { duration, or pain } \\
\text { catastrophizing). }\end{array}$ & $\begin{array}{l}11.1 \% \text { Overall } \\
\text { (5.6\% in tx vs. } \\
5.6 \% \text { in } \\
\text { control) }\end{array}$ \\
\hline
\end{tabular}

3-month follow up in

both tx conditions,

however NS in HA

frequency between

tx + control groups. 
Table 1 Description of studies meeting eligibility for the systematic review (Continued)

\begin{tabular}{|c|c|c|c|c|c|c|c|}
\hline \multirow[t]{2}{*}{$\begin{array}{l}\text { Multimodal } \\
\text { including CBT }\end{array}$} & \multirow[t]{2}{*}{$\mathrm{RCT}$} & \multirow[t]{2}{*}{$\begin{array}{l}\text { Hedborg, } \\
2012[50]\end{array}$} & \multirow[t]{2}{*}{$\begin{array}{l}N=76 \text { (ages } 22- \\
65) \text { Newspaper ads } \\
24 \text { weeks (MBT) }+ \\
36 \text { weeks (hand } \\
\text { massage) }\end{array}$} & \multirow[t]{2}{*}{$\begin{array}{l}\text { Migraine at least } \\
2 \text { times monthly }\end{array}$} & \multirow[t]{2}{*}{ Medication } & $\begin{array}{l}\text { - Decrease in total } \\
\text { migraine drug intake } \\
\text { at the end of the MBT } \\
\text { program in the MBT } \\
\text { group ( } 13.0 \text { vs. } 10.1 \\
\text { drug doses/subject/ } \\
56 \text { days) compared to } \\
\text { controls, no } \\
\text { significant difference } \\
\text { in total migraine } \\
\text { medication drug } \\
\text { intake in the control } \\
\text { group ( } 8.3 \text { vs. } 8.9 \text { drug } \\
\text { doses/subject/ } \\
56 \text { days) }\end{array}$ & \multirow[t]{2}{*}{$\begin{array}{l}8.4 \% \text { Overall } \\
\text { (7.4 \% in MBT } \\
\text { + hand } \\
\text { massage vs. } \\
14.3 \% \text { in MBT } \\
\text { vs. } 3.6 \% \text { in } \\
\text { control group) }\end{array}$} \\
\hline & & & & & & $\begin{array}{l}\text { - Drug efficacy } \\
\text { increased during MBT } \\
\text { from } 0.30 \text { to } 0.52 \\
(p<0.001) \text {, but this } \\
\text { was mainly explained } \\
\text { by the increase } \\
\text { proportion of mild HAs }\end{array}$ & \\
\hline \multirow[t]{2}{*}{$\begin{array}{l}\text { Multimodal } \\
\text { including CBT }\end{array}$} & \multirow[t]{2}{*}{$\mathrm{RCT}$} & \multirow[t]{2}{*}{$\begin{array}{l}\text { Hedborg, } \\
2011[51]\end{array}$} & \multirow[t]{2}{*}{$\begin{array}{l}N=83 \text { (ages 22- } \\
65 \text { ) Newspaper ads } \\
24 \text { weeks (MBT) }+ \\
36 \text { weeks (hand } \\
\text { massage) }\end{array}$} & \multirow[t]{2}{*}{$\begin{array}{l}\text { Migraine at least } \\
2 \text { times monthly }\end{array}$} & \multirow[t]{2}{*}{$\begin{array}{l}\text { HA frequency; } \\
\text { QoL; DAS; } \\
\text { Acceptability }\end{array}$} & $\begin{array}{l}\text { - } 40 \% \text { of patients } \\
\text { receiving MBT alone } \\
+42 \% \text { of patients } \\
\text { receiving MBT+ hand } \\
\text { massage had } 50 \%+ \\
\text { reduction in migraine } \\
\text { frequency when } \\
\text { compared to control } \\
\text { group. }\end{array}$ & \multirow[t]{2}{*}{$\begin{array}{l}8.4 \% \text { Overall } \\
\text { (7.4 \% in MBT } \\
+ \text { hand } \\
\text { massage vs. } \\
14.3 \% \text { in MBT } \\
\text { vs. } 3.6 \% \text { in } \\
\text { control group) }\end{array}$} \\
\hline & & & & & & $\begin{array}{l}\text { - Hand massage NS on } \\
\text { migraine frequency } \\
\text { compared to MBT } \\
\text { alone. }\end{array}$ & \\
\hline
\end{tabular}

- Frequency of HA

decreased

significantly from pre-

tx to post-tx in CBT

was significantly

group but not in con- CBT group but not in

trol (EDU) group.

control.

- NS difference

between the groups

in satisfaction or

"patient-therapist-

alliance/assistance"

oses/subject/

Drug efficacy

s mainly explaine 
Table 1 Description of studies meeting eligibility for the systematic review (Continued)

\begin{tabular}{|c|c|c|c|c|}
\hline $\begin{array}{l}\text { Multimodal } \\
\text { including } \\
\text { CBT + applied } \\
\text { relaxation }\end{array}$ & $\mathrm{RCT}$ & $\begin{array}{l}\text { Andersson, } \\
2003 \text { [38] }\end{array}$ & $\begin{array}{l}N=44 \text { (ages 18- } \\
\text { 59) Newspaper } \\
\text { ads, project } \\
\text { website } 6 \text { weeks }\end{array}$ & $\begin{array}{l}\text { Migraine, Tension- } \\
\text { type HA, or } \\
\text { cluster HA } \\
\text { (Dx = self-report) }\end{array}$ \\
\hline
\end{tabular}

relaxation

Multimodal

including

$\mathrm{PMR}+$

$\begin{array}{ll}\text { RCT (delayed } & \text { Devineni, } \\ \text { tx control } & 2005[52] \\ \text { which later } & \\ \text { crossed over) } & \end{array}$

$N=139$ (age not Migraine with or

specified) Internet- without aura

based promotion tension-type HA

channels e.g.

or mixed.

ads, websites

4 weeks
- NS in depression

(MADRS-S) scores

from baseline to post-

tx or across groups.

- Improvement in

"perceived work

performance" in hand

massage + MBT group

from baseline to al

follow-up points.

HA duration; HA . NS in HA frequency

index; HA days;

or intensity (either

- HA duration

decreased more in

$32 \%$ Overall

HA intensity; group or between

telephone arm

(29\% tx vs.

$(p<0.05)$

DAS

groups).

- NS in HI between

groups

- Self-reported

inventories (HADS

depression subscale,

HDI, PSS) showed

significant

improvements in

both groups but not

between groups.

HA duration; $\quad$ Only a non-

HA index; HA

frequency; HA

severity;

Medication index

Disability; DAS;

significant trend was

- There was a trend

towards a between

group difference in

found for \# of HA

medication index

days per week be-

post tx $(p=0.12)$

$38.1 \%$ Overall

$58.8 \%$ in

immediate tx

group vs.

$70.4 \%$ in

- There was a $\quad$.\% of tx completers

significant decrease in with clinically significan

delayed $t x$

$\begin{array}{ll}\text { significant decrease in } & \text { with clinically significant } \\ \text { peak intensity } & \text { improvement }(50 \%\end{array}$

between the $t x$ and decrease in $\mathrm{HI}$ ) was

control groups post-tx $38.5 \%$ vs. $6.4 \%$

(waitlist)

- Estimated time

expenditure for the

therapist $=1.3 \mathrm{~h} /$

participant $($ range $=$

$0.2-8.8 \mathrm{~h}$ ), resulting in

a cost-effectiveness

estimate of 0.32 .

- Greater compliance

was associated with

greater improvement

in primary $\mathrm{HA}$

outcomes. 
Table 1 Description of studies meeting eligibility for the systematic review (Continued)

\begin{tabular}{|c|c|c|c|c|c|c|c|c|}
\hline \multirow[t]{2}{*}{$\begin{array}{l}\text { Multimodal } \\
\text { including PMR }\end{array}$} & \multirow[t]{2}{*}{$\begin{array}{l}\text { Randomized } \\
\text { to intervention } \\
\text { vs waitlist }\end{array}$} & \multirow[t]{2}{*}{$\begin{array}{l}\text { Strom, } 2000 \\
{[53]}\end{array}$} & \multirow[t]{2}{*}{$\begin{array}{l}N=102 \text { (ages 19- } \\
62) \text { Newspaper } \\
\text { articles + Internet } \\
\text { magazines } \\
6 \text { weeks }\end{array}$} & \multirow[t]{2}{*}{$\begin{array}{l}\text { Recurrent HA } \\
(>6 \text { months, at } \\
\text { least } 1 \mathrm{HA} \text { per } \\
\text { week) }\end{array}$} & \multirow[t]{2}{*}{$\begin{array}{l}\text { HA duration; HA } \\
\text { index; HA days; } \\
\text { HA intensity; HA } \\
\text { severity; } \\
\text { Medication index; } \\
\text { Disability; DAS; } \\
\text { Cost }\end{array}$} & $\begin{array}{l}\text { - Decrease in HA } \\
\text { days + HA peak } \\
\text { intensity post-tx in } \\
\text { the tx group } \\
\text { compared to the } \\
\text { control group. }\end{array}$ & $\begin{array}{l}\text { - Improvement in } \mathrm{HI} \\
\text { (average reduction in } \\
\text { HI was } 31 \% \text { for tx } \\
\text { group vs. } 3 \% \text { for } \\
\text { control group, } \\
p=0.028 \text { ). }\end{array}$ & \multirow[t]{2}{*}{56 \% Overall } \\
\hline & & & & & & $\begin{array}{l}\text { - NS in Headache } \\
\text { Disability Inventory } \\
\text { (HDI) or Beck } \\
\text { Depression Inventory } \\
\text { (BDI). }\end{array}$ & $\begin{array}{l}\text { - Cost-effectiveness: } \\
\text { Estimated sum of } \\
\text { therapist time = } \\
40 \text { hrs/participant. } \\
\text { Cost-efficiency esti- } \\
\text { mate: } 0.78\end{array}$ & \\
\hline \multirow[t]{3}{*}{$\begin{array}{l}\text { Multimodal } \\
\text { including } \\
\text { relaxation }\end{array}$} & \multirow[t]{3}{*}{$\mathrm{RCT}$} & \multirow[t]{3}{*}{$\begin{array}{l}\text { Kleiboer, } \\
2014 \text { [54] }\end{array}$} & \multirow{3}{*}{$\begin{array}{l}N=368 \text { (ages 18- } \\
65 \text { ) HA specialist } \\
\text { referral, website, } \\
\text { flyers, newspaper/ } \\
\text { magazine ads } \\
8 \text { weeks- } \\
11.4 \text { weeks, } 8 \\
\text { lessons, to be } \\
\text { done in 7-10 days }\end{array}$} & \multirow[t]{3}{*}{$\begin{array}{l}\text { Migraine with or } \\
\text { without aura + } \\
2-6 \text { attacks/ } \\
30 \text { days prior to } \\
\text { randomization }\end{array}$} & \multirow[t]{3}{*}{$\begin{array}{l}\text { HA frequency; HA } \\
\text { days; HA severity; } \\
\text { Self-efficacy; QoL; } \\
\text { Disability; LoC }\end{array}$} & $\begin{array}{l}\text { - A } 20-25 \% \text { decrease } \\
\text { in migraine frequency } \\
\text { was found for both the } \\
\text { tx + control groups, NS } \\
\text { between groups. }\end{array}$ & \multirow{3}{*}{$\begin{array}{l}\text { - BT (tx group) had } \\
\text { significantly more } \\
\text { improvement that the } \\
\text { control group in } \\
\text { migraine-related self- } \\
\text { efficacy }(p<0.001 \text {, } \\
\mathrm{ES}=0.86),+ \text { devel- } \\
\text { oped more internal } \\
\text { control }(p, 0.001 \text {, ES }= \\
0.57) \text { but less external } \\
\text { control } \\
(p<0.001, E S=0.78) \text {. }\end{array}$} & \multirow[t]{3}{*}{$\begin{array}{l}27.4 \% \text { Overall } \\
\text { (39.0\% in tx } \\
\text { group vs. } \\
14.5 \% \text { in } \\
\text { control group) }\end{array}$} \\
\hline & & & & & & $\begin{array}{l}\text { - A significant but small } \\
\text { decrease in average } \\
\text { attack peak intensity } \\
\text { was seen in the ITT BT } \\
\text { (tx) group from } \\
\text { baseline to post-tx, but } \\
\text { NS between groups. }\end{array}$ & & \\
\hline & & & & & & $\begin{array}{l}\text { - Compliance was } \\
\text { explored in a random } \\
\text { sample of } 60 \\
\text { participants, which } \\
\text { showed that } \\
\text { participants reported } \\
\text { conducting at least } \\
\text { one relaxation exercise } \\
\text { on } 45.5 \% \text { of days of } \\
\text { being in training. }\end{array}$ & & \\
\hline \multirow[t]{2}{*}{ Other } & \multirow[t]{2}{*}{$\begin{array}{l}\text { Descriptive } \\
\text { study }\end{array}$} & \multirow[t]{2}{*}{$\begin{array}{l}\text { Sorbi, } 2010 \\
\text { [55] }\end{array}$} & \multirow[t]{2}{*}{$\begin{array}{l}N=10 \text { (ages 31- } \\
68 \text { ) } \\
\text { Individuals with } \\
\text { recently expressed } \\
\text { interest in self- } \\
\text { management } \\
\text { training } 10 \text { weeks }\end{array}$} & \multirow[t]{2}{*}{$\begin{array}{l}\text { Migraine with } \\
1-6 \text { attacks/ } \\
\text { month }\end{array}$} & \multirow[t]{2}{*}{ Acceptability } & & $\begin{array}{l}\text { - All lessons were } \\
\text { rated positively } \\
\text { regarding clarity, } \\
\text { instructiveness, } \\
\text { importance + easy } \\
\text { execution by new } \\
\text { participants }\end{array}$ & \multirow[t]{2}{*}{$\begin{array}{l}40 \% \text { Overall } \\
\text { (New } \\
\text { Participants) }+ \\
0 \% \text { overall } \\
\text { (Expert } \\
\text { Patients) }\end{array}$} \\
\hline & & & & & & & $\begin{array}{l}\text { - Expert patients } \\
\text { provided positive } \\
\text { ratings for the web } \\
\text { application, digital } \\
\text { support, + web- } \\
\text { adaptation of the } \\
\text { protocol. }\end{array}$ & \\
\hline
\end{tabular}


Table 1 Description of studies meeting eligibility for the systematic review (Continued)

\begin{tabular}{|c|c|c|c|c|c|c|c|c|c|}
\hline & \multirow[t]{3}{*}{$\begin{array}{l}\text { Self- } \\
\text { Management } \\
\text { Program }\end{array}$} & \multirow[t]{3}{*}{$\begin{array}{l}\text { Descriptive study } \\
\text { (Interviews + } \\
\text { concept mapping } \\
\text { to develop Web- } \\
\text { prototype + study } \\
\text { feasibility) }\end{array}$} & \multirow[t]{3}{*}{$\begin{array}{l}\text { Donovan, } \\
2013 \text { [56] }\end{array}$} & \multirow{3}{*}{$\begin{array}{l}N=12 \text { (ages } 12- \\
17, \text { adolescents), } 9 \\
\text { (ages } 30-55, \\
\text { caregivers) } 12 \\
\text { (adults, } \\
\text { clinicians)** } \\
\text { Newspaper ads + } \\
\text { community } \\
\text { message board } \\
\text { (adolescents + } \\
\text { caregivers). Emails/ } \\
\text { invitation at } \\
\text { conference } \\
\text { (clinicians) } 60 \text { min } \\
\text { (interview) + } \\
30 \text { min } \\
\text { (acceptance } \\
\text { testing via } \\
\text { telephone) }\end{array}$} & \multirow[t]{3}{*}{ Migraine } & \multirow[t]{3}{*}{ Acceptability } & & $\begin{array}{l}\text { - Disagreement over } \\
\text { content areas for the } \\
\text { website-clinicians but } \\
\text { not adolescents felt } \\
\text { diet + exercise were } \\
\text { important to include. }\end{array}$ & \multirow[t]{3}{*}{$\mathrm{N} / \mathrm{A}$} \\
\hline & & & & & & & & $\begin{array}{l}\text { - During the prototype } \\
\text { evaluation, most } \\
\text { adolescents indicated } \\
\text { that the website would } \\
\text { be useful (especially } \\
\text { the "Ask an Expert" } \\
\text { feature) when they felt } \\
\text { a migraine coming on } \\
\text { or had a migraine. }\end{array}$ & \\
\hline & & & & & & & & $\begin{array}{l}\text { - Caregivers reported } \\
\text { being "somewhat" to } \\
\text { "extremely likely" to } \\
\text { use the range of } \\
\text { features offered on } \\
\text { the website. }\end{array}$ & \\
\hline \multirow[t]{4}{*}{ PDA } & \multirow[t]{2}{*}{$\begin{array}{l}\text { Multimodal } \\
\text { including } \\
\text { relaxation }\end{array}$} & \multirow[t]{2}{*}{$\begin{array}{l}\text { Descriptive } \\
\text { Study + Case } \\
\text { Control study }\end{array}$} & \multirow[t]{2}{*}{$\begin{array}{l}\text { Kleiboer, } \\
2009[57]\end{array}$} & \multirow{2}{*}{$\begin{array}{l}N=44 \text { (ages 25- } \\
63)^{* * *} \mathrm{HA} \\
\text { websites, } \\
\text { newspaper ad, } \\
\text { referral by HA } \\
\text { specialists } 3 \text { weeks }\end{array}$} & \multirow[t]{2}{*}{ Migraine } & \multirow[t]{2}{*}{$\begin{array}{l}\text { HA frequency; } \\
\text { QoL; LoC; } \\
\text { Acceptability }\end{array}$} & \multirow{2}{*}{$\begin{array}{l}\text { - There were no } \\
\text { significant } \\
\text { improvements in HA } \\
\text { frequency in the } \\
\text { ODA + BT group (tx) } \\
\text { compared to BT alone } \\
\text { (control). }\end{array}$} & $\begin{array}{l}\text { - ODA was considered } \\
\text { feasible, well- } \\
\text { accepted + perceived } \\
\text { to support self-care. }\end{array}$ & \multirow[t]{2}{*}{$29.5 \%$ Overall } \\
\hline & & & & & & & & $\begin{array}{l}\text { - There were no } \\
\text { significant } \\
\text { improvements in } \\
\text { internal control or } \\
\text { migraine-specific QoL } \\
\text { in the ODA + BT group } \\
\text { compared to BT alone. }\end{array}$ & \\
\hline & \multirow[t]{2}{*}{ Other } & \multirow[t]{2}{*}{$\begin{array}{l}\text { Descriptive } \\
\text { pilot study } \\
\text { (To establish } \\
\text { feasibility) }\end{array}$} & \multirow[t]{2}{*}{$\begin{array}{l}\text { Sorbi, } 2007 \\
\text { [39] }\end{array}$} & \multirow[t]{2}{*}{$\begin{array}{l}N=5 \text { (ages } 24-52 \text { ) } \\
\text { Unknown } 8.5 \text { days } \\
\text { on average (range } \\
4-12 \text { days) }\end{array}$} & \multirow[t]{2}{*}{$\begin{array}{l}\text { Migraine without } \\
\text { aura }\end{array}$} & \multirow[t]{2}{*}{ Acceptability } & \multirow[t]{2}{*}{$\begin{array}{l}\text { - In the second run, } \\
\text { adherence was } 85 \% \text {. }\end{array}$} & $\begin{array}{l}\text { - ODA had good } \\
\text { acceptability } \\
\text { evidenced by positive } \\
\text { participant responses }\end{array}$ & \multirow[t]{2}{*}{$0 \%$ Overall } \\
\hline & & & & & & & & $\begin{array}{l}\text { - Loss of data due to } \\
\text { technical problems } \\
\text { amounted to } 6.8 \% \text { of } \\
\text { potential diary } \\
\text { entries + lost internet } \\
\text { connection } \\
\text { contributed to loss of } \\
5.6 \% \text { of lost diary } \\
\text { entries. }\end{array}$ & \\
\hline
\end{tabular}


Table 1 Description of studies meeting eligibility for the systematic review (Continued)

\begin{tabular}{|c|c|c|c|c|c|c|c|c|c|}
\hline \multirow[t]{8}{*}{ Other } & \multirow[t]{2}{*}{$\begin{array}{l}\text { Multimodal } \\
\text { including } \\
\text { biofeedback }\end{array}$} & \multirow[t]{2}{*}{$\begin{array}{l}\text { Prospective, } \\
\text { single-arm, } \\
\text { open-label } \\
\text { pilot study }\end{array}$} & \multirow[t]{2}{*}{$\begin{array}{l}\text { Shiri, } 2013 \\
{[58]}\end{array}$} & \multirow[t]{2}{*}{$\begin{array}{l}N=10 \text { (ages 10- } \\
17.5) \text { Pediatric } \\
\text { neurology clinic } \\
6 \text { months- } \\
60 \text { months }\end{array}$} & \multirow[t]{2}{*}{$\begin{array}{l}\text { Chronic migraine } \\
\text { or Chronic } \\
\text { Tension-type HA }\end{array}$} & \multirow[t]{2}{*}{$\begin{array}{l}\text { QoL; Activity } \\
\text { limitation; Other }\end{array}$} & \multirow{2}{*}{$\begin{array}{l}\text { - Patients reported a } \\
\text { decline in HA severity } \\
\text { (VAS } 4.28 \text { pre-test } \\
\text { vs. } 3.11 \text { post-test, } \\
\text { p } 0.015 \text { ) + signficant } \\
\text { improvements in daily } \\
\text { function + quality } \\
\text { of life. }\end{array}$} & $\begin{array}{l}\text { - Improvement pre-tx } \\
\text { to post-tx in quality of } \\
\text { life (Pedsi QL) + daily } \\
\text { function (measured } \\
\text { by } 2 \text { questions on } \\
\text { VAS scale) }\end{array}$ & \multirow[t]{2}{*}{$10 \%$ Overall } \\
\hline & & & & & & & & $\begin{array}{l}\text { - Overall the } \\
\text { participants reported } \\
\text { they were satisfied } \\
\text { with the tx. }\end{array}$ & \\
\hline & \multirow[t]{4}{*}{$\begin{array}{l}\text { Multimodal } \\
\text { including } \\
\text { Biofeedback }\end{array}$} & \multirow[t]{4}{*}{$\mathrm{RCT}$} & \multirow[t]{4}{*}{$\begin{array}{l}\text { Scharff, } \\
2002[59]\end{array}$} & \multirow[t]{4}{*}{$\begin{array}{l}N=36 \text { (ages 7-17) } \\
\text { Referred from } \\
\text { children's hospital } \\
6 \text { weeks }\end{array}$} & \multirow[t]{4}{*}{$\begin{array}{l}\text { Migraine with } \\
\text { or without aura } \\
\text { +/-co-existing } \\
\text { tension-type HA }\end{array}$} & \multirow[t]{4}{*}{$\begin{array}{l}\text { HA index; HA } \\
\text { days; HA severity; } \\
\text { DAS; Acceptability }\end{array}$} & $\begin{array}{l}\text { - Change in \# of HAs } \\
\text { recorded + highest } \\
\text { intensity rating over } \\
\text { time, but there were } \\
\text { no significant } \\
\text { between-group } \\
\text { differences. Likely due } \\
\text { to small } n+\text { low } \\
\text { power of the study. }\end{array}$ & \multirow{2}{*}{$\begin{array}{l}\text { - } 53.8 \% \text { (7) of children } \\
\text { in the handwarming } \\
\text { biofeedback group, } \\
10 \%(1) \text { in the } \\
\text { handcooling } \\
\text { biofeedback group, and } \\
0 \%(0) \text { in the waitlist } \\
\text { control group had a } \\
50 \% \text { or more decrease } \\
\text { in HI at the post tx. The } \\
\text { significantly higher } \\
\text { proportion of } \\
\text { participants achieving } \\
50 \% \text { reduction in HI in } \\
\text { handwarming group } \\
\text { vs. handcooling group } \\
\text { was maintained at } \\
3 \text { month }+6 \text { month } \\
\text { follow-up. }\end{array}$} & \multirow[t]{4}{*}{$\begin{array}{l}9.4 \% \text { Overall } \\
(0 \% \text { in } \\
\text { handwarming } \\
\text { group vs. } \\
9.1 \% \text { in } \\
\text { handcooling } \\
\text { group vs. } \\
8.3 \% \text { in WLC } \\
\text { group) }\end{array}$} \\
\hline & & & & & & & \multirow[t]{3}{*}{$\begin{array}{l}\text { - Adherence: Data } \\
\text { from home practice } \\
\text { records of } 29 \\
\text { participants in } \\
\text { handwarming or } \\
\text { handcooling group } \\
\text { indicated the average } \\
\text { \# of practice sessions } \\
\text { was } 5.3 \text { times per } \\
\text { week. }\end{array}$} & & \\
\hline & & & & & & & & $\begin{array}{l}\text { - NS in CDI or STAIC } \\
\text { scores. }\end{array}$ & \\
\hline & & & & & & & & $\begin{array}{l}\text { - There was a } \\
\text { temperature change } \\
\text { between the } \\
\text { handwarming + } \\
\text { handcooling groups, } \\
\text { with the handwarming } \\
\text { group more likely to } \\
\text { report that their } \\
\text { temperatures } \\
\text { increased. }\end{array}$ & \\
\hline & \multirow[t]{2}{*}{$\begin{array}{l}\text { Other-Sound } \\
\text { therapy }\end{array}$} & \multirow[t]{2}{*}{$\begin{array}{l}\text { RCT (double } \\
\text { blind, placebo- } \\
\text { controlled study } \\
\text { with a parallel } \\
\text { group add-on } \\
\text { design) }\end{array}$} & \multirow[t]{2}{*}{$\begin{array}{l}\text { Trinka, } \\
2002[60]\end{array}$} & \multirow[t]{2}{*}{$\begin{array}{l}N=32 \text { (ages } 16- \\
60) \text { Outpatient HA } \\
\text { clinic } 12 \text { weeks }\end{array}$} & \multirow[t]{2}{*}{$\begin{array}{l}\text { Migraine with or } \\
\text { without aura } \\
\text { assessed by } \\
\text { neurologists }\end{array}$} & \multirow{2}{*}{\multicolumn{2}{|c|}{$\begin{array}{l}\text { DAS; Acceptability; } \\
\text { Other }\end{array}$}} & $\begin{array}{l}\text { - Raw values of the } \\
\text { "headache" subtest of } \\
\text { the GBB improved in } \\
\text { both groups but NS } \\
\text { between groups. }\end{array}$ & \multirow[t]{2}{*}{$\begin{array}{l}\text { No Adherence } \\
\text { Data }\end{array}$} \\
\hline & & & & & & & & - NS in FPI-R, STAI or & \\
\hline
\end{tabular}


Table 1 Description of studies meeting eligibility for the systematic review (Continued)

\begin{tabular}{|c|c|c|c|c|c|c|c|c|}
\hline $\begin{array}{l}\text { PMR+ } \\
\text { Biofeedback }\end{array}$ & $\begin{array}{l}\text { Prospective non } \\
\text { randomized }\end{array}$ & $\begin{array}{l}\text { Arena, } \\
2004[61]\end{array}$ & $\begin{array}{l}N=4 \text { (ages 52-64) } \\
\text { Medical Center } \\
8 \text { weeks }\end{array}$ & $\begin{array}{l}\text { Migraine or } \\
\text { combined } \\
\text { migraine-tension } \\
\mathrm{HA}\end{array}$ & $\begin{array}{l}\text { HA index; HA } \\
\text { days; HA severity; } \\
\text { Medication index }\end{array}$ & $\begin{array}{l}\text { - } 1 \text { subj had } 50 \% \text { or } \\
\text { greater reduction in } \\
\mathrm{Hl}, 2 \text { had some } \\
\text { clinical improvement, } \\
1 \text { subject } \\
\text { demonstrated no tx } \\
\text { response }\end{array}$ & & $0 \%$ Overall \\
\hline \multirow[t]{2}{*}{ Biofeedback } & $\begin{array}{l}\text { Prospective non } \\
\text { randomized }\end{array}$ & $\begin{array}{l}\text { Folen, } \\
2001[62]\end{array}$ & $\begin{array}{l}N=\text { unknown**** } \\
\text { U.S Army/Navy } \\
\text { hospitals Not } \\
\text { specified }\end{array}$ & $\begin{array}{l}\text { Migraine, Chronic } \\
\text { daily HAs }\end{array}$ & Acceptability & & $\begin{array}{l}\text { - When evaluating the } \\
\text { viability of the system } \\
\text { in } 2 \text { separate rooms } \\
\text { of the medical center, } \\
\text { patient satisfaction } \\
\text { was high }(8 / 10)+ \\
\text { patients produced } \\
\text { physiologic changes } \\
\text { in desired direction. }\end{array}$ & $\begin{array}{l}\text { No adherence } \\
\text { data }\end{array}$ \\
\hline & & & & & & & $\begin{array}{l}\text { - Total cost of the } \\
\text { system about } \$ 9000\end{array}$ & \\
\hline
\end{tabular}

$*=8$ weeks ( 56 days) to 11.4 weeks ( 80 days) (=recommended duration of treatment. With 8 lessons, each lesson advised to be completed in 7-10 days) Actual average treatment duration $=3.6$ months (suggesting it took $\sim 2$ wks per lesson).** $=$ First group of adolescents/ caregivers for interviews and concept mapping: 12 (ages 12-17, adolescents), 9 (ages 30-55, caregivers) 12 (adults, clinicians).*The same procedure was used to recruit a second group of adolescents and their caregivers and clinicians to evaluate the prototype website: ?12? (ages 12-17, adolescents), ?9? (34-55, "mothers") and ?12? (clinicians, adults). *** =44 (ages 25-63) for ODA group feasibility and utility study aim, 31 (ages 25-59) in ODA+ BT group, 31 (ages 26-58) in ODA- group (matched controls). **** =N=not specified ("a number of patients") A description of 2 patients with headache who received biofeedback with the ProComp remote system was provided, however they indicate there are more patients who have received this treatment and state a study to objectively evaluate

equivalency between telehealth and in-vivo treatment is underway. HI, Headache Index; Tx, Treatment; HA, Headache; HA days, Days with headache; CPC, Chronic pain coping; DAS, Depression anxiety stress; QoL, Quality of life; LoC, Locus of control 
As indicated in Table 2, the outcomes measured from the various studies ranged considerably. About one third of studies $(7 / 23,30 \%)$ used measures of headache occurrence as outcomes such as a $50 \%$ reduction in headache frequency, headache intensity or headache index as an outcomes measure. The most frequent outcome assessed was acceptability of/satisfaction with the intervention, with 9/23 (39 \%) studies assessing this. Five studies (22\%) used medications or a medication index for assessment. 6/23 (26 \%) studies used measures of depression, anxiety and/or stress. Only 2/23 (9 \%) studies evaluated cost. Three (13\%) used self-efficacy and/or locus of control.

Many of the studies were considered "pilot" studies and thus had a small number of subjects, limiting the ability to detect differences between treatment groups and controls. 16/23 (70 \%) studies had a $N \geq 30$. Of the 16, one study by Bromberg et al. [27] had technical issues leading to the loss of data. Of the remaining 15 studies, six had statistically significant positive findings for their respective primary outcomes (frequency and/or intensity and/or disability and/or medications taken) in the electronic behavioral intervention group compared to controls. No studies addressed power analysis.

Looking at the $9 / 23$ (39 \%) studies that offered CBT interventions with $N \geq 30$, there was a positive outcome in headache frequency, severity, intensity and/or migraine medications taken in 4/9 (44 \%) studies. Of the $3 / 23(13 \%)$ studies which used biofeedback as part of the behavioral intervention, only two (67\%) had $N \geq 30$ and one (33\%) of these studies had a decrease in peak intensity. Of the $8 / 23$ (35\%) studies using relaxation, 7 (88 \%) had $N \geq 30$ and of these, only $2 / 7$ (29\%) had positive outcomes (frequency and peak intensity).

One study by Bromberg and colleagues evaluated whether there was a dose response by test determining whether more time spent on the intervention website resulted in a greater treatment effect. The researcher divided the treatment group into low-dose and high-dose groups. Participants who used the site the most reported greater increases in feelings of self-efficacy from baseline to post-intervention $(t=5.41$, $P<.0010), 3$ months $(t=4.53, P<.0010)$ and 6 months $(t=4.64, P<.0010)$ but outcome variables aside from self-efficacy were not affected [27]. This study also performed a completer analysis (i.e. whether there was a different intervention effect for participants who completed all study assessments compared with those who did not). For all but 2 outcomes (self-efficacy and headache locus of control), no difference in treatment effect was noted for completers compared with noncompleters.

\section{Discussion}

Of the 23 papers on digitally delivered behavioral interventions for headache, all were published after the year 2000 and most (55\%) were published after the year 2010. Overall results suggest moderate to high rates of acceptability and feasibility of using technology to treat headaches, although efficacy data are more limited. Patients were comfortable using a variety of delivery modalities including CD-ROMs in two studies, the internet in 14 studies, personal digital assistants in two studies, custom biofeedback devices in three studies, and a personal audio player in one study. Although the pilot nature of many of these studies makes it difficult to directly compare outcomes, results of these 23 studies can help guide future efforts to develop more effective, engaging, and evidence-based digital interventions for headache.

Focusing on outcomes, results of the 23 studies suggest that patients are willing to use digitallydelivered behavioral headache interventions. The studies used varied headache related outcome measures: headache index, headache duration, headache frequency, headache intensity, medications, self-efficacy, quality of life, sleep, anxiety, depression, distress, and disability. Also, the studies used many diverse outcome measures regarding the use of the technology being studied: satisfaction, feasibility, tolerability, adherence, and engagement among others. The variability in reporting instruments used to record headache symptoms limits direct comparison between studies. However, in an effort to broadly understand the overall impact on headache, we considered which interventions led to a $50 \%$ or greater reduction in headache symptoms seen only in the intervention and not in the control group. Only a minority of studies were able to demonstrate this statistically significant reduction in symptoms. The combination of internet based interventions with CBT had the most positive effect on headache reduction. Given the widespread acceptance of the effectiveness of CBT for headache symptoms and the increasing access as well as comfort that many have with the internet, the successful combination is not surprising. Internet-delivered CBT has also been an area of intense research in other fields such as psychiatry in exploring applications for treating depression [28-30] and in pain medicine for reducing discomfort. [31-33] Along these lines, given the high prevalence of psychiatric comorbidity and migraine [34], future studies can be done to explore whether these electronic behavioral interventions help pain outcomes and the psychiatric symptoms.

The results of many of the reviewed studies must be understood in terms of several common themes. Dropout rates for the digital interventions ranged widely. 
Table 2 Outcomes table ([27, 38-57] 58-62)

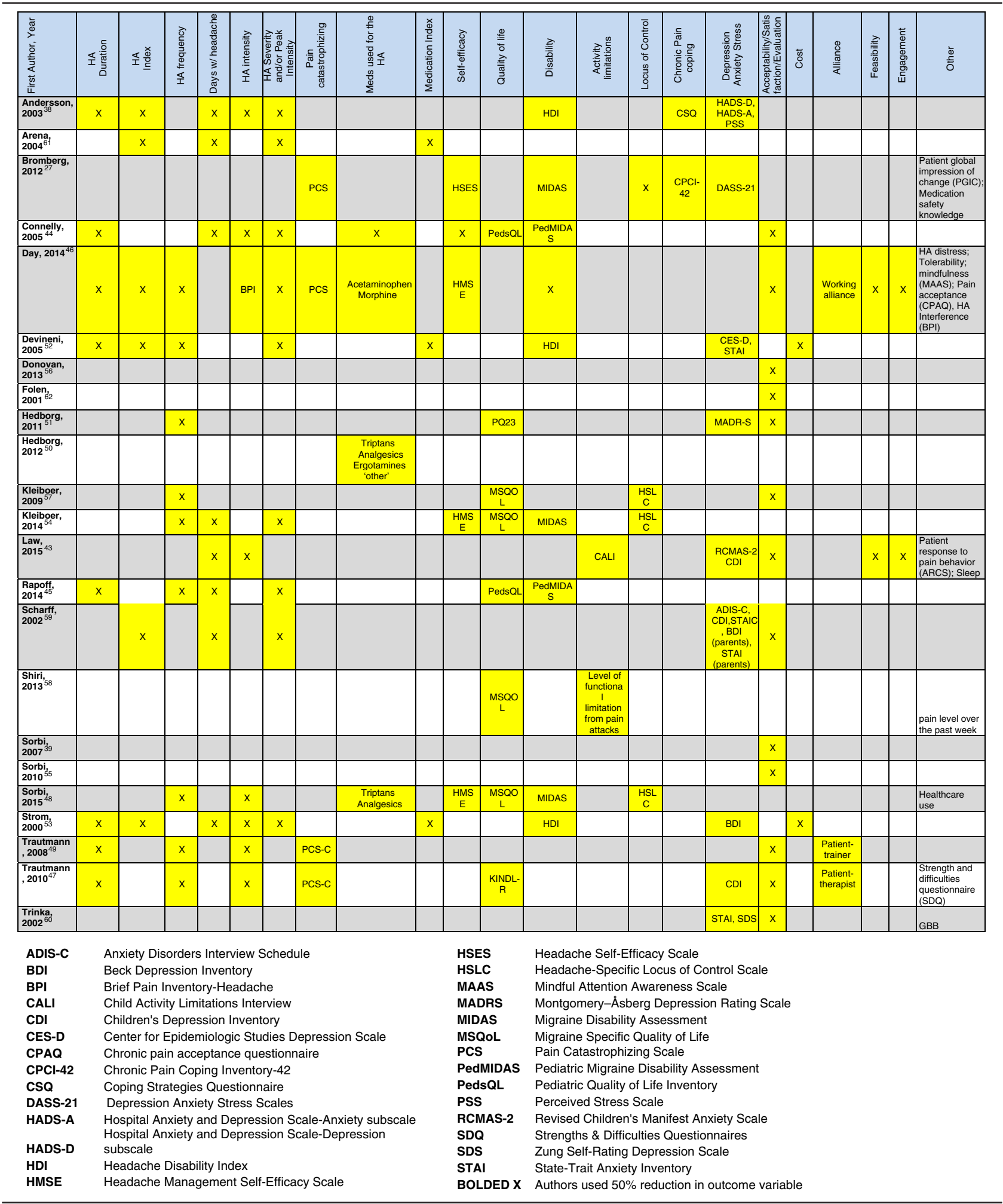


Adherence is a common concern for headache medication [35, 36], with estimates that anywhere from $25 \%$ to $94 \%$ of adults adhere to headache treatment regimens [35]. Thus, adherence is also a concern for digital interventions. Recent studies of computer based CBT delivered for purposes outside of headache have suggested that the average user may only complete a single session of computer based CBT [37] and our review suggests the same issue may occur when using computer CBT for headache. These adherence rates in the review may actually be higher than in clinical practice as some studies tied compensation to adherence and others offered personal check-ins or other study staff interventions to encourage adherence. Given the chronic nature of many headache conditions, long term adherence or adherence with booster sessions may be important if digital interventions are to have long term efficacy. The average duration of studies was only 6 weeks, rendering it difficult to fully understand how engaged patients will be with digital tools that may be used over a period of months instead of weeks. Future studies can be done to better assess adherence. Future studies can evaluate the need for booster sessions to help maintain skills. Engagement of patients, families, and treating clinicians in the development, refinement, and post study iterative improvement in the intervention would likely increase adherence and potency of the treatment. Studies can also be done to determine whether the psychiatric comorbidities such as anxiety and depression play a role in adherence with electronic behavioral treatments for headache. In addition, whether these evidence-based treatments can effectively be delivered electronically outside of the clinical setting remains to be seen.

Beyond concerns regarding adherence, understanding the impact of these digitally delivered behavioral interventions for headache is difficult because of the numerous confounding variables in many of the individual studies. But rather than being just a cause for caution, these confounding variables also suggest important research agenda items in seeking to understand the mechanism and efficacy of these interventions. For example, numerous studies involved human contact, be it in the form of a research assistant checking in to a therapist offering personalized feedback, although the impact of such additional human interaction and feedback was hardly explored. In our review, we found one study conducted to explicitly determine the role of such human contact; the study by Andersson and colleagues examined whether telephone contact by a therapist improved adherence and headache related disability, stress, and coping outcomes when used with an internet-based cognitive behavioral self-help program. However, therapist-initiated telephone calls did not influence the results [38]. Also, many of the interventions employed technologies that prompted use between once a day to several times per day but again the impact and optimal number of prompts was examined in only one study, in which the investigators noted that users displayed some annoyance about the number of digital prompts they received [39]. Along similar lines, no studies looked at the right "dose" of behavioral interventions, e.g., how many CBT sessions are necessary, the most effective length of each CBT session, and if and when should there be refresher lessons. Of the reviewed papers, the average number of sessions or lessons was six and was static and fixed for each study. Finally, few studies employed blinded controls (e.g., sham CBT) making it hard to understand the true placebo effect of receiving what may be perceived as 'high tech treatment.' However, per the Behavioral Clinical Headache Trials Guidelines, the placebo is not a realistic expectation of behavioral trials anyway [40].

In this era of increasing interest in mobile health, smartphone apps, and wearables, it was interesting to note the paucity of literature exploring the mobile delivery of behavioral interventions for headache. This is especially interesting as smartphones can fulfill many of the delivery modalities explored in the literature (cd-rom, internet, personal digital assistants, audio) and also offer all the interventions offered in the literature (CBT, self-management, relaxation, and biofeedback). With ownership of smartphones in the general population at $64 \%$ in 2015 [41] and expected to increase in 2016, it seems likely that smartphones will may offer an accessible and practical tool for behavioral interventions for treatment of headache. However, further data is needed on those who do not have access or ability to use a smartphone in order to understand the true potential. Still, the lack of any outcomes literature on this topic reflects a critical opportunity of researchers.

Despite the paucity of data on smartphone interventions, a quick search of the Apple or Android app marketplaces shows that this lack of a clinical evidence base has not stopped industry or consumer interest in headache intervention apps. Recent reviews have called into question the quality of these headache apps on the commercial marketplaces [42]. Assuming that the same complexities of efficacy and adherence that were noted in the 14 studies of internet delivered CBT for headache may also be applicable for smartphone delivered interventions-such concerns about the utility of commercially based headache apps seem valid although more research is needed here.

Several limitations must be considered in light of our methodology. As a literature review, we only report on published clinical studies and ignore industry 
and private efforts to use technology to deliver behavioral interventions for headache. While it is likely that industry efforts have achieved results beyond those noted in our review-validating such technologies in clinical studies remains critical. Also, we did not evaluate the quality of CBT, biofeedback or PMR delivered in the reviewed papers and it is possible that differences in the delivery of the behavioral intervention itself may have impacted outcomes. For example, one study used not individual but family based CBT [43]. In addition, we did not control for study methodology with some studies offering subjects more encouragement and assistance which may have also impacted adherence rates. Quantifying such support is difficult as many studies were vague or did not report on exact amount or quantity of assistance provided in using the technology. Finally, we acknowledge that there are no established data on the effect sizes of the electronic behavioral interventions for headache. We used $N \geq 30$ to collectively describe studies that were not just very small pilot studies.

\section{Conclusion}

Through this systematic review we have explored the literature on digitally delivered behavioral interventions for headache. While results suggest feasibility, data on efficacy and adherence is harder to interpret. The lack of studies on smartphones is notable and presents an important research opportunity going forward.

\section{Appendix}

\section{Search strategy}

PUBMED, Embase and Psycinfo, 2000-December 11, 2015, restricted to English

The search strategy was as follows:

("Headache"[Mesh] OR "Headache Disorders"[Mesh] OR "Headache Disorders, Primary" $[\mathrm{MeSH}] \mathrm{OR}$ "Migraine Disorders" $[\mathrm{MeSH}]$ OR “Tension-Type Headache"[Mesh] OR headache[tiab] OR headaches[tiab] OR migraine[tiab] OR migraines[tiab] OR migranous[tiab] OR "head pain"[tiab] OR "head pains"[tiab] OR Cephalalgia[tiab] OR Cephalalgias[tiab] OR "Cranial Pains"[tiab] OR "Cranial Pain"[tiab] OR "tension-type headache"[tiab] OR "tension-type headaches"[tiab] OR "tension headache" [tiab] OR "tension headaches"[tiab] OR headache[ot] OR headaches[ot] OR migraine[ot] OR migraines[ot] OR "head pain" [ot] OR Cephalalgia[ot] OR Cephalalgias[ot] OR "Cranial Pain" [ot] OR "tension-type headache" [ot] OR "tension-type headaches" [ot] OR "tension headache"[ot]) AND ("Mobile Applications"[Mesh] OR "Internet"[Mesh] OR "Telemedicine"[Mesh] OR telemedicine[tiab] OR "Telephone"[Mesh] OR telephone[tiab] OR “Computers"[Mesh] OR "audiovisual
aids"[Mesh] OR “mp3-player"[Mesh] OR “Therapy, Computer-Assisted"[Mesh] "electronics, medical"[Mesh] OR "Electronics" [Mesh] OR "software" [MeSH] OR "online systems" [MeSH] OR "cell phones" [MeSH] OR smartphone[tiab] OR "smart phone"[tiab] OR iphone[tiab] OR mobile[tiab] OR cellphone[tiab] OR cellphones[tiab] OR "cell phone"[tiab] OR "cell phones"[tiab] OR internet[tiab] OR mhealth[tiab] OR "mobile health"[tiab] OR telehealth[tiab] OR eHealth[tiab] OR computer[tiab] OR computers[tiab] OR electronic[tiab] OR electronics[tiab] OR "medical electronics" [tiab] OR "world wide web" [tiab] OR "web based"[tiab] OR device[tiab] OR devices[tiab] OR technology[tiab] OR technologies[tiab] OR tech[tiab] OR "remote delivery" [tiab] OR "remotely delivered"[tiab] OR "mp3 player" [tiab] OR ipod[tiab] OR iPad[tiab] OR online[tiab] OR "communication technology"[tiab] OR wireless[tiab] OR "medical informatics"[Mesh] OR digital[tiab] OR online[ot] OR smartphone[ot] OR "smart phone"[ot] OR iphone[ot] OR mobile[ot] OR cellphone[ot] OR cellphones[ot] OR "cell phone"[ot] OR "cell phones"[ot] OR internet[ot] OR mhealth[ot] OR "mobile health"[ot] OR telehealth[ot] OR eHealth[ot] OR computer[ot] OR computers[ot] OR electronic[ot] OR electronics[ot] OR "medical electronics" [ot] OR "world wide web" [ot] OR "web-based"[ot] OR device[ot] OR devices[ot] OR technology[ot] OR technologies[ot] OR tech[ot] OR "mp3 player"[ot] OR ipod[ot] OR iPad[ot] OR online[ot] OR wireless[ot] OR telephone[ot] OR "telephone administered"[tiab]) AND ("Behavior Therapy"[Mesh] OR "Biofeedback, Psychology"[Mesh] OR "Desensitization, Psychologic"[Mesh] OR "relaxation therapy"[Mesh] OR "neurofeedback"[Mesh] OR "MindBody Therapies"[Mesh] OR "Cognitive Therapy"[Mesh] OR "meditation"[Mesh] OR "muscle relaxation"[Mesh] OR "self care" $[\mathrm{MeSH}]$ OR "behavior therapy"[tiab] OR "behaviour therapy"[tiab] OR "behavior therapies"[tiab] OR "behaviour therapies" OR "behavioral therapies"[tiab] OR "behavioural therapies"[tiab] OR "behavioral treatment"[tiab] OR "behavioural treatment"[tiab] OR "behavioral treatments"[tiab] OR "behavior treatment"[tiab] OR "behavioral approach"[tiab] OR "behavioral approaches"[tiab] OR biofeedback[tiab] OR biofeedbacks[tiab] OR neurofeedback[tiab] OR "cognitive therapy"[tiab] OR "cognitive therapies"[tiab] OR "cognitive behavioral therapy" [tiab] OR "cognitive behavioral treatment"[tiab] OR "cognitive behavioral treatments" OR "cognitive behavioral therapies"[tiab] OR "Cognitivebehavioral interventions"[tiab] OR "Cognitive-behavioral intervention"[tiab] OR "Psychologic Desensitization"[tiab] OR "relaxation techniques"[tiab] OR "relaxation technique"[tiab] OR "relaxation therapy" [tiab] OR "relaxation therapies"[tiab] OR "guided relaxation"[tiab] OR meditation[tiab] OR meditate[tiab] OR "Progressive muscle relaxation"[tiab] OR "neurofeedback"[tiab] OR 
"guided imagery"[tiab] OR "music therapy"[tiab] OR "mind-body therapy" [tiab] OR "mind-body therapies" [tiab] OR behavioral training [tiab] OR self-management [tiab] OR self-care [ot] OR psychological therapy [tiab] OR psychological therapies [tiab] OR "psychosocial support"[tiab] OR "behavior therapy"[ot] OR "behaviour therapy"[ot] OR "behavior therapies"[ot] OR "behaviour therapies" OR "behavioral therapies"[ot] OR "behavioural therapies"[ot] OR "behavioral treatment"[ot] OR "behavioural treatment" [ot] OR "behavioral treatments"[ot] OR "behavior treatment"[ot] OR "behavioral approach"[ot] OR "behavioral approache$\mathrm{s}$ "[ot] OR biofeedback[ot] OR neurofeedback[ot] OR "cognitive therapy"[ot] OR "cognitive therapies"[ot]
OR "cognitive behavioral therapy" [ot] OR "cognitive behavioral treatment"[ot] OR "cognitive behavioral treatments" OR "cognitive behavioral therapies" [ot] OR "Cognitive-behavioral interventions"[ot] OR "Cognitivebehavioral intervention" [ot] OR "relaxation techniques"[ot] OR "relaxation technique" [ot] OR "relaxation therapy"[ot] OR "guided relaxation"[ot] OR meditation[ot] OR meditate[ot] OR "Progressive muscle relaxation"[ot] OR "neurofeedback"[ot] OR "guided imagery" [ot] OR "music therapy" [ot] OR "mind-body therapy"[ot] OR "mind-body therapies"[ot] OR behavioral training [ot] OR self-management [ot] OR selfcare[ot] OR psychological therapy[ot] OR psychological therapies[ot])

Table 3 Various descriptions of cognitive behavioral therapy (CBT) conducted in the studies

Connelly, 2005[44]

Rapoff, 2014 [45]

Day, 2014 [46]

Bromberg, 2012 [27]

Law, 2015 [43]

Trautmann, 2010 [47]

Sorbi, 2015 [48]

Trautmann, 2008 [49]

Hedborg, $2012[50,51]$

\section{A CD-ROM program was provided to give additional strategies to help manage head pain.}

"...lessons on how to use various empirically supported cognitive-behavioral treatments to self-manage recurrent headaches...focused on problem-solving and stress management, and targeted pain behavior and parental response to pain."

"8-week MBCT for depression Protocol" Protocol: Segal Z, Williams JM, Teasdale J. Mindfulness-based Cognitive Therapy for Depression: A New Approach to Preventing Relapse. New York: The Guilford Press; 2002.

"Lessons-interactive instruction for learning practical pain self-management skills and strategies, and how to apply them to solve problems. Tools—visual and graphic interactive learning experiences that allow users to actively manipulate information to construct knowledge and learn to solve problems. Self-assessments-structured sets of questions that help users to reflect on and learn what skills and knowledge they have, where their relative strengths and deficits are, and how to identify what behaviors to target for change."

"Psychological therapy included face-to-face cognitive behavioral therapy for pain management and/or biofeedback. In addition, participants received access to an Internet CBT program (Web-based Management of Adolescent Pain; Web-MAP). The design and treatment content of Web-MAP was identical to the original version of the program." Original program: Palermo TM, Wilson AC, Peters M, Lewandowski A, Somhegyi H. Randomized controlled trial of an internet-delivered family cognitive-behavioral therapy intervention for children and adolescents with chronic pain. Pain. 2009;146:205-213.

"Adapted from the manualized face-to-face group therapy program devised by Denecke and Kro" ner-Herwig for children with recurrent headache." Manual in German: Denecke, H., \& Kroner-Herwig, B. (2000). Kopfschmerztherapie mit Kindern und Jugendlichen. Ein Trainingsprogramm.Gottingen: Hogrefe. "The first module presented education on headaches, the second unit focused on stress management (perception of own stress symptoms, coping with stress). In the following modules the participants acquired various skills including "cognitive restructuring (identification of dysfunctional cognitions regarding headache and stress and identifying functional cognitions)."

Focused on "graded tasks, specific goal setting and review of behavioral goals; behavior self-monitoring with teaching the use of prompts and cues, feedback on performance and barrier identification; behavioral modeling and social comparison, a focus on time- or stress-management, and relapse prevention. Adapted from several studies and later on turned into a module: Sorbi MJ and Swaen SJ. Protocollaire behandeling van patienten met migraine en spanningshoofdpijn:Ontspannings training en cognitieve training (Training according to protocol in migraine and tension-type headache: Relaxation training and cognitive training). In: Keijsers GPJ, van Minnen A and Hoogduin CAL(eds) Protocollaire behandelingen in de ambulante geestelijke gezondheidszorg (Treatment according to protocol in ambulatory mental health care). Houten: Bohn Stafleuvan Loghum, 2004, pp.219-260.

6 self-help sessions (focusing on education on headaches, stress management, relaxation, cognitive restructuring, self-assurance strategies, problem solving) based on a face-to-face training manual. Manual: Kroner-Herwig, B. and Denecke, H. (2002). Cognitive-behavioural therapy of paediatric headache. Are there any differences in efficacy between a therapist-administered group training and a self-help format? Journal of Psychosomatic Research, $53,1107-1114$.

53-page training program aimed at improving stress coping skills and divided into the following topics: stress physiology, physical activity, diet, thought patterns, handling of emotions, and attitudes. Training Program: Hedborg K and Muhr C. Multimodal behavioral treatment of migraine: an Internet-administered, randomized, controlled trial. Ups J Med Sci 2011; 116: 169-186. 


\section{Competing interests}

Dr. Richard Lipton reports grants and personal fees from Alder, personal fees from Allergan, personal fees from CoLucid, grants and personal fees from Electrocore, personal fees and other from eNeura, personal fees from Ethicon, personal fees from Merck, grants and personal fees from Novartis, personal fees from Labrys, personal fees from Autonomic Technologies, personal fees from Boston Scientific, personal fees from Bristol Myers Squibb, personal fees from Dr. Reddys, personal fees from Eli Lilly, personal fees from Endo Pharmaceuticals, personal fees from Informa, grants from Migraine Research Fund, grants from National Headache Foundaiton, grants from $\mathrm{NIH}$, personal fees from Teva, personal fees from Vedanta, outside the submitted work.

Dr. Mary Ann Sevick's mentoring effort is covered under an NIH-K24NR01226 award.

\section{Authors' contributions}

MTM conceived of the study, participated in the design and coordination of the systematic review and helped to draft the manuscript. JT conceived of the study, participated in the design and coordination of the systematic review and helped to draft the manuscript. JR helped to acquire and analyze the data and helped to draft the manuscript. AP helped to acquire and analyze the data and helped to draft the manuscript. CG participated in the conception of the systematic review and revised it critically for important intellectual content. SP helped with the interpretation of the data and critically reviewed the draft for important intellectual content. RL helped with the interpretation of the data and critically reviewed the draft for important intellectual content. MS RL helped with the interpretation of the data and critically reviewed the draft for important intellectual content. All authors read and approved the final manuscript.

\section{Author details}

'Department of Neurology, NYU Langone Medical Center, 240 East 38th Street 20th floor, New York, NY 10016, USA. NYU Langone Headache Center, Department of Neurology, NYU School of Medicine, New York, NY, USA. ${ }^{3}$ Department of Psychiatry, Beth Israel Deaconess Medical Center and Brigham and Women's Hospital, Harvard Medical School, Boston, MA, USA. ${ }^{4}$ NYU School of Medicine, New York, USA. ${ }^{5}$ Department of Library Services, NYU School of Medicine, New York, USA. ${ }^{6}$ Department of Emergency Medicine, NYU Langone Medical Center, NYU School of Medicine, New York, NY, USA. ${ }^{7}$ Cincinnati Children's Medical Center, Headache Center, Office for Clinical and Translational Research, Center for Child Behavior and Nutrition Research and Training, Pediatrics Cincinnati, Cincinnati, Ohio, USA. ${ }^{8}$ Montefiore Headache Center, Department of Neurology, Albert Einstein College of Medicine, Neurology, Bronx, USA. ${ }^{9}$ Center for Behavioral Change, Department of Population Health, NYU School of Medicine, New York, NY, USA.

\section{Received: 20 January 2016 Accepted: 17 February 2016}

\section{Published online: 10 May 2016}

\section{References}

1. Penzien DB, Rains JC, Lipchik GL, Nicholson RA, Lake AE 3rd, Hursey KG (2005) Future directions in behavioral headache research: Applications for an evolving health care environment. Headache 45(5):526-534

2. Silberstein SD (2000) Practice parameter: Evidence-based guidelines for migraine headache (an evidence-based review): Report of the quality standards subcommittee of the American Academy of Neurology. Neurology 55(6):754-762

3. Campbell J, Penzien D, Wall E (1999) Evidence-based guidelines for migraine headache: Behavioral and physical treatments

4. Andrasik F, Blanchard EB, Neff DF, Rodichok LD (1984) Biofeedback and relaxation training for chronic headache: A controlled comparison of booster treatments and regular contacts for long-term maintenance. J Consult Clin Psychol 52(4):609-615

5. Schafer AM, Rains JC, Penzien DB, Groban L, Smitherman TA, Houle TT (2011) Direct costs of preventive headache treatments: Comparison of behavioral and pharmacologic approaches. Headache 51(6):985-991. doi:10.1111/j.1526-4610.2011.01905.x

6. Mobile technology fact sheet. Pew Research Center Web site. http://www. pewinternet.org/fact-sheets/mobile-technology-fact-sheet/. Updated 2014. Accessed 1/8, 2016.
7. Mobile medical applications. http://www.fda.gov/MedicalDevices/ DigitalHealth/MobileMedicalApplications/default.htm. Updated 9/22/15. Accessed 12/23, 2015.

8. Research to guidance. Research to Guidance Web site. http://www. research2guidance.com/500m-people-will. Updated 2015. Accessed 12/23, 2015.

9. McGillicuddy J, Weiland A, Frenzel R et al (2013) Patient attitudes toward mobile phone-based health monitoring: Questionnaire study among kidney transplant recipients. J Med Internet Res 15(1):e6

10. Blake H (2008) Innovation in practice: Mobile phone technology in patient care. Br J Community Nurs 13(4):160

11. Robinson E, Higgs S, Daley A et al. (2013) Development and feasibility testing of a smart phone based attentive eating intervention. BMC Public Health 13:639.

12. Worringham C, Rojek A, Stewart I (2011) Development and feasibility of a smartphone, ECG and GPS based system for remotely monitoring exercise in cardiac rehabilitation. PLoS One 6(2):e14669

13. Nes A, van Dulmen S, Eide E et al (2012) The development and feasibility of a web-based intervention with diaries and situational feedback via smartphone to support self-management in patients with diabetes type 2. Diabetes Res Clin Pract 97(3):385

14. Mark T, Fortner B, Johnson G (2008) Evaluation of a tablet PC technology to screen and educate oncology patients. Support Care Cancer 16(4):371

15. Dennison L, Morrison L, Conway G, Yardley L (2013) Opportunities and challenges for smartphone applications in supporting health behavior change: Qualitative study. J Med Internet Res 15(4):e86

16. Lyles C, Harris L, Le T et al (2011) Qualitative evaluation of a mobile phone and web-based collaborative care intervention for patients with type 2 diabetes. Diabetes Techno Ther 13(5):563

17. Rabin C, Bock B (2011) Desired features of smartphone applications promoting physical activity. Telemed J E Health 17(10):801

18. Huguet A, Stinson J, MacKay B et al (2014) Bringing psychosocial support to headache sufferers using information and communication technology: Lessons learned from asking potential users what they want. Pain Res Manag 19:e1-e8

19. Payne H, Lister C, West J, Bernhardt J (2015) Behavioral functionality of mobile apps in health interventions: A systematic review of the literature. JMIR mHealth uHealth 3(1):e20

20. Giffin NJ, Ruggiero L, Lipton RB et al (2003) Premonitory symptoms in migraine: An electronic diary study. Neurology 60(6):935-940

21. Ganser AL, Raymond SA, Pearson JD. Data quality and power in clinical trials: a comparison of ePRO and paper in a randomized trial. In: Byrom B, Tiplady B, editors. ePRO: electronic solutions for patient-reporteddata. Surray: Gower; 2010. pp. 49-78.

22. Dale O, Hagen KB (2007) Despite technical problems personal digital assistants outperform pen and paper when collecting patient diary data. J Clin Epidemiol 60(1):8-17

23. Greenwood MC, Hakim AJ, Carson E, Doyle DV (2006) Touch-screen computer systems in the rheumatology clinic offer a reliable and userfriendly means of collecting quality-of-life and outcome data from patients with rheumatoid arthritis. Rheumatology (Oxford) 45(1):66-71

24. Jose N, Langel K. ePRO vs. paper. Applied Clinical Trials. 2010.

25. Mia Minen, John Torous, Allison Piazza. Systematic review of behavioral interventions delivered electronically for headache. PROSPERO 2015: CRD42015032284 Available from: http://www.crd.york.ac.uk/PROSPERO/ display_record.asp?ID=CRD42015032284.

26. Moher D, Liberati A, Tetzlaff J, Altman D, The PRISMA group (2009) Preferred reporting items for systematic reviews and meta-analyses: The PRISMA statement. PLoS One 6(6):e1000097

27. Bromberg J, Wood ME, Black RA, Surette DA, Zacharoff KL, Chiauzzi EJ (2012) A randomized trial of a web-based intervention to improve migraine self-management and coping. Headache 52:244-261

28. Arnberg FK, Linton SJ, Hultcrantz M, Heintz E, Jonsson U (2014) Internetdelivered psychological treatments for mood and anxiety disorders: A systematic review of their efficacy, safety, and cost-effectiveness. PLoS One 9(5):1-13. doi:10.1371/journal.pone.0098118

29. Hedman E, Ljotsson B, Lindefors N (2012) Cognitive behavior therapy via the internet: A systematic review of applications, clinical efficacy and costeffectiveness. Expert Rev Pharmacoecon Outcomes Res 12(6):745-764. doi:10.1586/erp.12.67 
30. Vallury KD, Jones M, Oosterbroek C (2015) Computerized cognitive behavior therapy for anxiety and depression in rural areas: A systematic review. J Med Internet Res 17(6):e139. doi:10.2196/jmir.4145

31. Palermo TM, Wilson AC, Peters M, Lewandowski A, Somhegyi H (2009) Randomized controlled trial of an internet-delivered family cognitivebehavioral therapy intervention for children and adolescents with chronic pain. Pain 146:205-213

32. Fisher E, Law E, Palermo TM, Eccleston C (2015) Psychological therapies (remotely delivered) for the management of chronic and recurrent pain in children and adolescents. Cochrane Database Syst Rev 3:Cd011118

33. Eccleston C, Fisher E, Craig L, Duggan GB, Rosser BA, Keogh E (2014) Psychological therapies (internet-delivered) for the management of chronic pain in adults. Cochrane Database Syst Rev 2:CD010152

34. Minen MT, Begasse De Dhaem O, Kroon Van Diest A et al (2016) Migraine and its psychiatric comorbidities. J Neurol Neurosurg Psychiatry

35. Ramsey RR, Ryan JL, Hershey AD, Powers SW, Aylward BS, Hommel KA (2014) Treatment adherence in patients with headache: A systematic review. Headache 54(5):795-816. doi:10.1111/head.12353

36. Seng EK, Rains JA, Nicholson RA, Lipton RB (2015) Improving medication adherence in migraine treatment. Curr Pain Headache Rep 19(6):24-015-0498-8. doi:10.1007/s11916-015-0498-8

37. Gillbody S, Littlewood E, Hewitt C, et al (2015) Computerised cognitive behaviour therapy (CCBT) as treatment for depression in primary care (REEACT trial): Large scale pragmatic randomised controlled trial. BMJ 351(h5627).Epub ahead of print

38. Andersson G, Lundstrom P, Strom L (2003) Internet-based treatment of headache: Does telephone contact add anything? Headache 43:353-361

39. Sorbi MJ, Mak SB, Houtveen JH, Kleiboer AM, van Doornen L (2007) Mobile web-based monitoring and coaching: Feasibility in chronic migraine. J Med Internet Res 9(5):e38. doi:10.2196/jmir.9.5.e38

40. Penzien DB, Andrasik F, Freidenberg BM et al (2005) Guidelines for trials of behavioral treatments for recurrent headache, first edition: American headache society behavioral clinical trials workgroup. Headache 45(Suppl 2):S110-32

41. Smith A. Chapter one: A portrait of smartphone ownership. Pew Research Center Web site. http://www.pewinternet.org/2015/04/01/chapter-one-aportrait-of-smartphone-ownership/. Published April 1, 2015. Updated 2015. Accessed 1/8, 2016.

42. Hundert A, Huguet A, McGrath P, Stinson J, Wheaton M (2014) Commercially available mobile phone headache diary apps: A systematic review. JMR MHealth UHealth 2(3):e36

43. Law EF, Beals-Erickson SE, Noel M, Claar R, Palermo TM (2015) Pilot randomized controlled trial of internet-delivered cognitive-behavioral treatment for pediatric headache. Headache 55(10):1410-25

44. Connelly MA, Rapoff MA, Thompson N, Connelly W (2006) 'Headstrong': A computer-based cognitive-behavioral intervention for recurrent pediatric headache. J Pediatr Psychol 31(7):737-47.

45. Rapoff MA, Connelly M, Bickel JL et al (2014) Headstrong intervention for pediatric migraine headache: A randomized clinical trial. J Headache Pain 15:12

46. Day MA, Thorn BE, Ward L et al (2014) Mindfulness-based cognitive therapy for the treatment of headache pain: A pilot study. Clin J Pain 30:152-161

47. Trautmann E, Kroner-Herwig B (2010) A randomized controlled trial of internet-based self-help training for recurrent headache in childhood and adolescence. Behav Res Ther 48:28-37

48. Sorbi M, Kleiboer A, van Silfhout H, Vink G, Passchier J (2015) Medium-term effectiveness of online behavioral training in migraine self-management: A randomized trial controlled over 10 months. Cephalalgia 35(7):608-18.

49. Trautmann E, Kroner-Herwig B (2008) Internet-based self-help training for children and adolescents with recurrent headache: A pilot study. Behavioural and Cognitive Psychotherapy 36:241-245

50. Hedborg K, Muhr C (2012) The influence of multimodal behavioral treatment on the consumption of acute migraine drugs: A randomized, controlled study. Cephalalgia 32:297-307

51. Hedborg K, Muhr C (2011) Multimodal behavioral treatment of migraine: An internet-administered, randomized, controlled trial. Ups J Med Sci 116:169-186

52. Devineni T, Blanchard EB (2005) A randomized controlled trial of an internet-based treatment for chronic headache. Behav Res Ther 43:277-292
53. Strom L, Pettersson R, Andersson G (2000) A controlled trial of self-help treatment of recurrent headache conducted via the internet. J Consult Clin Psychol 68:722-727

54. Kleiboer A, Sorbi M, van Silfhout M, Kooistra L, Passchier J (2014) Short-term effectiveness of an online behavioral training in migraine self-management: A randomized controlled trial. Behav Res Ther 61:61-69

55. Sorbi MJ, van der Vaart R (2010) User acceptance of an internet training aid for migraine self-management. J Telemed Telecare 16:20-24

56. Donovan E, Mehringer S, Zeltzer LK (2013) Assessing the feasibility of a web-based self-management program for adolescents with migraines and their caregivers. Clin Pediatr (Phila) 52:667-670

57. Kleiboer A, Sorbi M, Merelle S, Passchier J, van Doornen L (2009) Utility and preliminary effects of online digital assistance (ODA) for behavioral attack prevention in migraine. Telemed J E Health 15:682-690

58. Shiri $S$, Feintuch $U$, Weiss $N$, et al. A virtual reality system combined with biofeedback for treating pediatric chronic headache-A pilot study. Pain Med. 2013;14(5):621-7.

59. Scharff L, Marcus DA, Masek BJ. A controlled study of minimal-contact thermal biofeedback treatment in children with migraine. J Pediatr Psychol. 2002;27(2):109-19.

60. Trinka E, Unterrainer J, Luthringshausen $\mathrm{G}$, et al. An auditory electrophysiological intervention in migraine: A randomized placebo controlled add on trial. Journal of Neurotherapy 2002;6(2):21-30.

61. Arena J, Dennis N, Devineni T, Maclean R, Meador K. A pilot study of feasibility and efficacy of telemedicine-delivered psychophysiological treatment for vascular headache. Telemed J E Health. 2004;10:449-454.

62. Folen RA, James LC, Earles JE, Andrasik F. Biofeedback via telehealth: A new frontier for applied psychophysiology. Appl Psychophysiol Biofeedback. 2001;26:195-204.

\section{Submit your manuscript to a SpringerOpen ${ }^{\circ}$ journal and benefit from:}

- Convenient online submission

- Rigorous peer review

- Immediate publication on acceptance

- Open access: articles freely available online

- High visibility within the field

- Retaining the copyright to your article

Submit your next manuscript at springeropen.com 